\title{
Infinite Dimensional Dynamical Systems and the Navier-Stokes Equation
}

\author{
C. Eugene Wayne* \\ Department of Mathematics and Statistics \\ Boston University
}

August 2, 2007

\begin{abstract}
In this set of lectures I will describe how one can use ideas of dynamical systems theory to give a quite complete picture of the long time asymptotics of solutions of the two-dimensional Navier-Stokes equation. I will discuss the existence and properties of invariant manifolds for dynamical systems defined on Banach spaces and review the theory of Lyapunov functions, again concentrating on the aspects of the theory most relevant to infinite dimensional dynamics. I will then explain how one can apply both of these techniques to the two-dimensional Navier-Stokes equation to prove that any solution with integrable initial vorticity will will be asymptotic to a single, explicitly computable solution known as an Oseen vortex. equations.
\end{abstract}

${ }^{*}$ The work of the author is supported in part by the NSF under grant number DMS-0405724 


\section{Lecture 1: Infinite dimensional dynamical systems}

In this first lecture I recall some common techniques used in finite dimensional dynamical systems and discuss their generalization to the infinite dimensional context needed for applications to partial differential equations. The two main tools we will use in these lectures will by invariant manifolds and Lyapunov functions. We will use the former to analyze the behavior of systems near stationary solutions and the latter to obtain more global information about solutions. Good general references for this material are [11] and [12].

We begin by recalling a very simple situation. Suppose that one has a system of $n$ ordinary differential equations

$$
\frac{d \mathbf{x}}{d t}=\tilde{f}(\mathbf{x}), \quad \mathbf{x} \in \mathbb{R}^{n} .
$$

Suppose further that the origin is a fixed point of this this system of equations. If we want to analyze the behavior of solutions near zero an obvious approach is to linearize the equation i.e. we write

$$
\tilde{f}(\mathbf{x})=\tilde{f}(0)+\left(D_{0} \tilde{f}\right) \mathbf{x}+\mathcal{O}\left(|\mathbf{x}|^{2}\right) \equiv L \mathbf{x}+f(\mathbf{x})
$$

In this last equality we have used the fact that $\tilde{f}(0)=0$ ( and defined the $n \times n$ matrix $L=D_{0} \tilde{f}$ - i.e. the Jacobian matrix of $\tilde{f}$ at the fixed point. The function $f(x)$ collects the nonlinear terms in the equation - in particular, $f(\mathbf{x})=\mathcal{O}\left(|\mathbf{x}|^{2}\right)$ for $\mathbf{x}$ near zero. If $\mathbf{x}$ is very small then the terms $\mathcal{O}\left(|\mathbf{x}|^{2}\right)$ should be much smaller than the linear terms in $\mathbf{x}$ suggesting that a good approximation to the solutions of (1) should be given by

$$
\frac{d \mathbf{x}}{d t}=L \mathbf{x}
$$

This equation is easily solved - if $L$ has $n$ linearly independent eigenvectors $\left\{\mathbf{v}_{j}\right\}_{j=1}^{n}$, with eigenvalues $\{\lambda\}_{j=1}^{n}$, then any solution of (3) can be written as

$$
\mathbf{x}(t)=c_{1} e^{\lambda_{1} t} \mathbf{v}_{1}+\cdots+c_{n} e^{\lambda_{n} t} \mathbf{v}_{n}
$$

for some choice of constants $c_{j}$.

Remark 1.1 The constants $c_{j}$ are determined by the initial conditions. If $\left\{\mathbf{w}_{j}\right\}_{j=1}^{n}$ are the adjoint-eigenvectors of $L$, normalized so that $\left\langle\mathbf{w}_{j}, \mathbf{v}_{k}\right\rangle=\delta_{j, k}$ then we have

$$
c_{j}=\left\langle\mathbf{w}_{j}, \mathbf{x}(0)\right\rangle \text {. }
$$

$\left(\right.$ Here $\langle\cdot, \cdot\rangle$ is the inner product on $\mathbb{R}^{n}$.) Hence for later use we note that we will want to know not only eigenvectors for the linear part of equations we study but also adjoint eigenvectors.

From (4) we see that we can split $\mathbb{R}^{n}$ into a direct sum of three subspaces - the stable subspace, $\mathbb{E}^{s}$, the center subspace $\mathbb{E}^{c}$ and the unstable subspace, $\mathbb{E}^{u}$, which are respectively the spectral subspaces associated with the eigenvalues whose real parts have negative, zero, 
or positive real parts. Note that any solution with initial condition in $\mathbb{E}^{s}$ approaches the origin as $t \rightarrow \infty$ while any solution with initial condition in $\mathbb{E}^{u}$ approaches the origin as $t \rightarrow-\infty$.

An obvious question is to what extent this structure survives when we include the nonlinear terms that were omitted in (3). We certainly don't have explicit solutions like those in (4) any longer but geometrical structures analogous to the stable, center and unstable subspaces do persist, at least in a neighborhood of the fixed point - this is the content of the invariant manifold theorems. We state these informally for the moment, reserving a more formal treatment until we discuss the corresponding results for infinite dimensional systems below. Suppose $\tilde{f} \in C^{1}\left(\mathbb{R}^{n}\right)$. Then there exists a neighborhood of the origin $B_{r} \subset \mathbb{R}^{n}$ and a function $h^{s}$ defined on $B_{r}$ such that

$$
h^{s}: B_{r} \cap \mathbb{E}^{s} \rightarrow \mathbb{E}^{c} \oplus \mathbb{E}^{u}
$$

The function $h^{s}$ is $C^{1}$, and its graph, known as the local stable manifold $\mathcal{W}_{\text {loc }}^{s}$ is locally invariant (i.e. for any initial condition in $\mathcal{W}_{l o c}^{s}$ the corresponding solution of (1) remains in $\mathcal{W}_{l o c}^{s}$ for as long as it remains in the domain of definition of $h^{s}$.). Furthermore, any solution which remains in $\mathcal{W}^{s}$ for all $t \geq 0$ approaches the origin as $t \rightarrow \infty$. In addition the local stable manifold is locally unique - no other manifold in a neighborhood of the origin shares all these properties.

Analogous results hold for the local unstable manifold. However, things are slightly more delicate for the center-manifold (the manifold tangent at the origin to $\mathbb{E}^{c}$.) Although the existence of the center manifold follows much as for the stable and unstable manifolds, in general, the center manifold is not unique. There are simple examples of systems or ordinary differential equations with infinitely many local center manifolds.

One property that makes center manifolds particularly important and interesting is that one can show that there exists a neighborhood of the origin (which we can assume to be $B_{r}$, without loss of generality) such that any solution which remains in this neighborhood for all $t \in \mathbb{R}$ must lie in the local center manifold. This implies that any periodic orbits or additional fixed points in a neighborhood of the origin must lie in the center-manifold. If one is looking specifically for periodic orbits, say, this can lead to a very big simplification since it permits one to reduce the search from the original system of $n$ equations to a system whose dimension equals that of the center manifold which is often much less than $n$. Such a reduction is even more important in the context of partial differential equations where it frequently results in reduction from an infinite dimensional set of equations to one whose dimension is small and finite.

We next turn to a discussion of the appropriate generalization of these invariant manifold theorems to partial differential equations. Suppose that we consider a (system) of partial differential equations

$$
\frac{\partial u}{\partial t}=\mathcal{L} u+f(u, \nabla u)
$$

where $u=u(x, t) \in \mathbb{R}^{n}, x \in \mathcal{D} \subset \mathbb{R}^{d}$ and $t \geq 0 . \mathcal{L}$ is a linear, differential operator and $f$ is a nonlinear term depending on $u$ and its (first order) partial derivatives. One could also 
consider quasilinear partial differential equations but in these lectures we restrict attention to this semilinear case.

Following the intuition gained from the finite dimensional case above we would like to compare solutions of this equation to those of the linear equation

$$
\frac{\partial u}{\partial t}=\mathcal{L} u
$$

There are many additional difficulties that are encountered in treating this infinite dimensional case in comparison with the finite dimensional case discussed above. Some of these difficulties are only technical and reflect the more complicated analysis necessary in an infinite dimensional setting. However, other problems represent qualitative differences in the behavior of the partial differential equations vis-a-vis ordinary differential equations. Among the problems that must be overcome are:

1. The spectrum of $\mathcal{L}$ may no longer consist only of eigenvalues as in (3) but may now contain continuous spectrum.

2. Since the operator $\mathcal{L}$ will in general be unbounded it may not be possible to define solutions for $t<0$ for general initial conditions - in this case discussing the behavior of solutions as $t \rightarrow-\infty$ is clearly problematic!

3. If the continuous spectrum approaches the imaginary axis there may be not clear splitting between the center subspace and the stable and unstable subspaces. This problem, often called the lack of a spectral gap, is particularly common when studying problems defined on unbounded spatial domains.

4. One can't in general hope that the nonlinearity $f$ in (5) will be $C^{1}$ - indeed due to the presence of derivatives of $u$ in the nonlinear term it often even fails to map the Banach space in which solutions lie back into itself. This will be the case, for example in the Navier-Stokes equations which are the subject of the third and fourth lectures in this series.

Many authors have addressed the question of the existence and properties of invariant manifolds for partial differential equations. In contrast to the case of ordinary differential equations where it is more or less clear what the "right" assumptions on the vectorfield are and what the "correct" conclusion ought to be, this is by no means so clear in the case of partial differential equations. In particular, depending on the context one may wish to make either stronger or weaker assumptions about the linear part of the equation (which affect, for instance, the smoothing properties of the semi-group associated with (6), or even whether the linear part defines a semi-group). These assumptions then entail making either different assumptions on the nonlinear term, or changing (typically, weakening) the results one hopes to obtain. For examples of typical results in this context see [1], [13] or [16]. One general principle which emerges from this collection of results is that if (5) and (6) define semi-flows 
then it is often easier to work with the semi-flow than with the differential equation itself. This is because the semi-flow already incorporates any smoothing properties that the equation may possess. By working with the semi-flow, Chen, Hale and Tan (CHT) [4] have given a very general form of the invariant manifold theorem, applicable to many partial differential equations. It is their result that I will use in subsequent lectures and which I now state.

From now on, we assume that the partial differential equation (5) defines a semi-flow $\Phi^{t}$ on some Banach space $X$. Then (CHT) make the following assumptions:

(H.1) $\Phi^{t}(u)$ is continuous for $(t, u)$ in $\mathbb{R}^{+} \times X$ and there exist positive constants $q$ and $D$ such that

$$
\sup _{0 \leq t \leq q} \operatorname{Lip}\left(\Phi^{t}\right)=D<\infty
$$

where

$$
\operatorname{Lip}\left(\Phi^{t}\right) \equiv \sup _{u, v \in X} \frac{\left\|\Phi^{t}(u)-\Phi^{t}(v)\right\|}{\|u-v\|}
$$

(H.2) For some $\tau \in(0, q]$, one can decompose $\Phi^{\tau}$ as

$$
\Phi^{\tau}=S+R
$$

where $S$ is a bounded linear operator from $X$ to itself and $R$ is globally Lipshitz.

(H.3) There exist subspaces $X_{1}$ and $X_{2}$ such that $X=X_{1} \oplus X_{2}$, and continuous projections $P_{i}: X_{i} \rightarrow X_{i}, i=1,2$ which are invariant with respect to $S$. Also $S$ commutes with $P_{i}$. If $S_{i}=\left.S\right|_{X_{i}}$, then $S_{1}$ has bounded inverse and there exist constants $C_{i}$ and $\alpha_{i}$ such that $\alpha_{1}>\alpha_{2} \geq 0$ and

$$
\begin{aligned}
\left\|S_{1}^{-k} P_{1}\right\| & \leq C_{1} \alpha_{1}^{-k} \\
\left\|S_{2}^{k} P_{2}\right\| & \leq C_{2} \alpha^{k}
\end{aligned}
$$

(H.4) The constant $C_{i}$ and $\alpha_{i}$ from (H.3) are related to the nonlinearity in such a way that

$$
\left(\frac{\left(\sqrt{C_{1}}+\sqrt{C_{2}}\right)^{2}}{\alpha_{1}-\alpha_{2}}\right) \operatorname{Lip}(R)<1 .
$$

Remark 1.2 For later use we fix two additional constants $\gamma_{1}$ and $\gamma_{2}$ with $\alpha_{2}<\gamma_{2}<\gamma_{1}<\alpha_{1}$ such that

$$
\operatorname{Lip}(R)\left(\frac{C_{1}}{\alpha_{1}-\gamma_{1}}+\frac{C_{2}}{\gamma_{2}-\alpha_{2}}\right)=1
$$

Note that by making $\operatorname{Lip}(R)$ sufficiently small we can make $\gamma_{1}$ arbitrarily close to $\alpha_{1}$ and $\gamma_{2}$ arbitrarily close to $\alpha_{2}$. 
Remark 1.3 Before stating the conclusions of the (CHT) theorem we comment briefly on the meaning of these hypotheses. The hypothesis (H.1) implies that (5) defines a well-behaved semi-flow. This hypothesis typically rules out applying these results to elliptic equations, for example. Hypothesis (H.2) is just an assumption that the semi-group splits nicely into its linear and nonlinear parts. Hypothesis (H.3) implies a "spectral gap" for the linear part of the semigroup. The spectrum of $S_{2}$ must lie inside a circle of radius $\alpha_{2}$ and the spectrum of $S_{1}$ must lie outside a circle of radius $\alpha_{1}$. Note however, that there is no assumption that $S_{2}$ is invertible - we do not assume that the original partial differential equation is solvable "backwards" in time for general initial data. Finally, hypothesis (H.4) requires that the nonlinear term must be small, in the appropriate sense, with respect to the spectral gap.

We now state the theorem of (CHT) which we will use later:

Theorem 1.4 Suppose that (H.1)-(H.4) hold. Then there exists a globally Lipshitz map $g: X_{1} \rightarrow X_{2}$ such that the graph of $g$

$$
G=\left\{u_{1}+g\left(u_{1}\right) \mid u_{1} \in X_{1}\right\}
$$

satisfies:

(i) (Invariant Manifold) The restriction of $\Phi^{t}$ to $G$ can be extended to a Lipshitz flow on $G$.

(ii) (Lyapunov exponents) Any negative semi-orbit $\|u(t)\|_{t \leq 0} \subset X$ that satisfies

$$
\lim _{t \rightarrow \infty} \frac{1}{|t|} \log \|u(t)\|<-\frac{1}{\tau} \log \gamma_{1}
$$

must be contained in $G$. In particular, if $\gamma_{1}<1$, any fixed point of $\Phi^{t}$ must lie in $G$.

(iii) (Invariant Foliation) There exists a continuous map $h: X \times X_{2} \rightarrow X_{1}$ such that if $v \in G$, then $h\left(v, P_{2} v\right)=P_{1} v$ and the set

$$
M_{v}=\left\{h(v, w)+w \mid w \in X_{2}\right\}
$$

passing through $v$ satisfies $\Phi^{t}\left(M_{v}\right) \subset M_{\Phi^{t}(v)}$ and

$$
M_{v}=\left\{w \in X\left|\limsup _{t \rightarrow \infty} \frac{1}{t} \log \right| \Phi^{t}(w)-\Phi^{t}(v) \mid \leq \frac{1}{\tau} \log \gamma_{2}\right\}
$$

Remark 1.5 We didn't discuss the finite dimensional analogue of point (iii) but roughly speaking the fibers $M_{v}$ of this foliation contain all points whose asymptotic behavior it the same as that $v$ - i.e. we can characterize the asymptotics of all points (sufficiently close to the fixed point) by those of points on the invariant manifold. Note that the estimate on the rate of convergence of points in the fiber toward the invariant manifold also implies as a corollary that all solutions near the invariant manifold approach it (assuming that $\gamma_{2}<1$, as will be the case in the examples we consider.) 
Remark 1.6 Note that in Hypothesis (H.1) we assume that there is a global bound on the Lipshitz constant of the semi-flow. (Here, I mean global in X, not in time.) This is rarely true in practice but this hypothesis is why the manifold constructed here is not constrained to a neighborhood of the fixed point but rather is defined for all $u_{1} \in X_{1}$. In practice we "cut off" the nonlinear terms in the equation outside a small neighborhood of the fixed point in order to allow this hypothesis to be verified and this will make the applications of this theorem "local" in character.

Remark 1.7 If the term $R$ in the decomposition of the semiflow is nonlinear in the sense that $R(0)=0$ and $D R(0)=0$ then the function $g$ whose graph defines the invariant manifold has the same property - namely $g(0)=0$ and $D g(0)=0$.

This invariant manifold theorem will be our main tool to investigate the local behavior of solutions of partial differential equations in the later lectures. However we will also want to consider more global questions. For those, we will make use of Lyapunov functions. Here, the transition from the finite dimensional to infinite dimensional setting involves fewer changes than in the case of the invariant manifold theorems so we work directly with the infinite dimensional case without first reviewing the finite dimensional results. The presentation here largely follows that of D. Henry in [11] - see that work, or [12] for more details.

Let $\Phi^{t}$ be a semi-flow on a Banach space $X$. We want to characterize the long-time behavior of solutions of the differential equation defining $\Phi^{t}$ and with that in mind make the following two definitions:

Definition 1.8 Given $\mathbf{u}_{0} \in X$, we define the forward orbit of $\mathbf{u}_{0}$ as:

$$
\mathcal{O}^{+}\left(\mathbf{u}_{0}\right)=\left\{\Phi^{t}\left(\mathbf{u}_{0}\right) \mid t \geq 0\right\}
$$

Definition 1.9 The omega limit set of a point $\mathbf{u}_{0}$ is the set of all points which the forward orbit of $\mathbf{u}_{0}$ approaches arbitrarily closely as $t$ tends to infinity. More precisely,

$\omega\left(\mathbf{u}_{0}\right)=\left\{\mathbf{u} \in X \mid\right.$ there exists $\left\{t_{n}\right\} \subset \mathbb{R}$ such that $\lim _{n \rightarrow \infty} t_{n}=\infty$ and $\left.\lim _{n \rightarrow \infty}\left\|\Phi^{t_{n}}\left(\mathbf{u}_{0}\right)-\mathbf{u}\right\|=0\right\}$.

Exercise 1.10 Suppose that $\tilde{\mathbf{u}} \in \mathcal{O}^{+}\left(\mathbf{u}_{0}\right)$. Show that $\omega\left(\mathbf{u}_{0}\right)=\omega(\tilde{\mathbf{u}})$. Thus we can refer without ambiguity not just to $\omega\left(\mathbf{u}_{0}\right)$ but also $\omega\left(\mathcal{O}^{+}\left(\mathbf{u}_{0}\right)\right)$

Exercise 1.11 Show that if $\mathbf{u}^{*}$ is an element of the $\omega$-limit set of $\mathbf{u}_{0}$ then the orbit of $\mathbf{u}^{*}$ is defined for all $t \in \mathbb{R}$ and the entire orbit of $\mathbf{u}^{*}$ is contained in $\omega\left(\mathbf{u}_{0}\right)$.

One might worry that the omega-limit set was empty, but this turns out not to be the case, at least not if the forward orbit remains in a compact set: 
Proposition 1.12 If $\mathcal{O}^{+}\left(\mathbf{u}_{0}\right) \subset K$, a compact subset of $X$. then $\omega\left(\mathbf{u}_{0}\right)$ is non-empty and invariant (i.e. if $\mathbf{u}^{*} \in \omega\left(\mathbf{u}_{0}\right)$, then $\Phi^{t}\left(\mathbf{u}^{*}\right) \in \omega\left(\mathbf{u}_{0}\right)$ for all $t \in \mathbb{R}$.)

The proof of this proposition is not difficult - see [11] for details. The only slightly surprising point is that the omega-limit set is invariant in both forward and backward time, even though we don't know (or expect) that the semi-group itself is defined for $t \leq 0$ for general initial conditions.

A key tool for investigating omega-limit sets are Lyapunov functions.

Definition 1.13 If $X$ is a Banach space, a Lyapunov function for the semi-flow $\Phi^{t}$ is a continuous, real-valued function $\Psi$ such that

$$
\limsup _{t \rightarrow 0^{+}} \frac{\Psi\left(\Phi^{t}(\mathbf{u})\right)-\Psi(\mathbf{u})}{t} \leq 0 \text { for all } \mathbf{u} \in X .
$$

This means that $\Psi$ is non-increasing along orbits of $\Phi^{t}$.

Remark 1.14 Note that if the limit in Definition 1.13 exists it is just the derivative of $\Psi$ along the trajectory with initial condition $\mathbf{u}$ so a common way of verifying that a given function is a Lyapunov function is to show that its derivative is non-positive along solutions.

A key tool we will use in Lecture 4 is the LaSalle Invariance Principle:

Proposition 1.15 Let $\Psi$ be a Lyapunov function for the semi-flow $\Phi^{t}$. Define $\mathbb{E}=\{\mathbf{u} \in$ $\left.X\left|\frac{d}{d t} \Psi \circ \Phi^{t}(\mathbf{u})\right|_{t=0}=0\right\}$ If $\mathcal{O}^{+}\left(\mathbf{u}_{0}\right)$ is contained in a compact subset of $X$ then $\omega\left(\mathbf{u}_{0}\right) \subset \mathbb{E}$.

Because of the importance of this result for our applications we sketch its proof:

Proof: By the compactness of the forward orbit and continuity of $\Psi$ we know that there exists some finite $M$ such that

$$
V\left(\Phi^{t}\left(\mathbf{u}_{0}\right)\right) \geq M
$$

for all $t \geq 0$. Since $\Psi$ is monotonic along the orbit of $\mathbf{u}_{0}$ we therefore conclude that

$$
\lim _{t \rightarrow \infty} \Psi\left(\Phi^{t}\left(\mathbf{u}_{0}\right)\right)=\Psi^{\infty}
$$

for some $\Psi^{\infty}$. If $\mathbf{w} \in \omega\left(\mathbf{u}_{0}\right)$ the definition of the omega-limit set, plus the continuity of $\Psi$ imply that there exists a sequence of times $\left\{t_{n}\right\}$ approaching infinity such that $\lim _{n \rightarrow \infty} \Psi\left(\Phi^{t_{n}}\left(\mathbf{u}_{0}\right)\right)=$ $\Phi(\mathbf{w})$, from which we conclude that $\Phi(\mathbf{w})=\Psi^{\infty}$. But then, by the invariance of the omegalimit set we have

$$
\Psi\left(\Phi^{t}(\mathbf{w})\right)=\Psi^{\infty} \text { for all } t \in \mathbb{R},
$$

which implies that $\mathbf{w} \in \mathbb{E}$. 
Example 1.16 We finish this lecture with an example of a somewhat unusual Lyapunov function which will play a role later in this series of talks. Consider the dynamical system defined by the partial differential equation

$$
\begin{aligned}
& \frac{\partial w}{\partial \tau}= \mathcal{L} w, w=w(\xi, \tau) ; \xi \in \mathbb{R}^{d}, \tau \geq 0 \\
& w(\xi, 0)=w_{0}(\xi)
\end{aligned}
$$

where $\mathcal{L} w=\Delta_{\xi} w+\frac{1}{2} \nabla \cdot(\xi w)$. The reason for considering this unusual equation will be explained in Lecture 2 but for the moment assume two facts about the evolution:

(1.) The solutions of (8) obey the maximum principle. In particular, if $w_{0}(\xi) \geq 0$ then $w(\xi, \tau)>0$ for all $\xi$ for any $\tau>0$.

(2.) If $w(\xi, 0) \in L^{1}\left(\mathbb{R}^{d}\right)$ the $\omega$-limit set of the corresponding trajectory exists.

The reason that (8) obeys the maximum principle will be explained in Lecture 2.

We next show that the $L^{1}$ norm is a Lyapunov function .

Lemma 1.17 Let $w_{0} \in L^{1}\left(\mathbb{R}^{d}\right)$ and let $w(\xi, \tau)$ be the solution of (8) with this initial condition. Then the function

$$
\Phi(w)(\tau)=\int_{\mathbb{R}^{d}}|w(\xi, \tau)| d \xi
$$

is non-increasing along trajectories. More precisely, $\Phi(w)(\tau) \leq \Phi\left(w_{0}\right)$ for every $\tau>0$ and equality holds if and only if $w_{0}$ does not change sign.

Proof: Define $w_{0}^{+}(\xi)=\max \left(w_{0}(\xi), 0\right), w_{0}^{-}(\xi)=-\min \left(w_{0}(\xi), 0\right)$ Let $w^{ \pm}(\xi, \tau)$ be the solutions of (8) with initial conditions $w_{0}^{ \pm}$respectively. Note that from the form of the equation we see immediately that the equation conserves the integral of the solution. Thus

$$
\int_{\mathbb{R}^{d}} w(\xi, \tau) d \xi=\int_{\mathbb{R}^{d}} w_{0}(\xi) d \xi
$$

and

$$
\int_{\mathbb{R}^{d}} w^{ \pm}(\xi, \tau) d \xi=\int_{\mathbb{R}^{d}} w_{0}^{ \pm}(\xi) d \xi
$$

Now note that if $w_{0}$ does change sign, $w_{0}^{ \pm}$are both non-trivial. Furthermore they have disjoint support. However, by the maximum principle, $w^{ \pm}(\xi, \tau)$ will both be positive for all $\xi$ whenever $\tau>0$. Thus,

$$
\begin{aligned}
\int_{\mathbb{R}^{d}} \mid & w(\xi, \tau)\left|d \xi=\int_{\mathbb{R}^{d}}\right| w^{+}(\xi, \tau)-w^{-}(\xi, \tau) \mid d \xi \\
& <\int_{\mathbb{R}^{d}}\left(w^{+}(\xi, \tau)+w^{-}(\xi, \tau)\right) d \xi=\int_{\mathbb{R}^{d}}\left(w_{0}^{+}(\xi)+w_{0}^{-}(\xi)\right) d \xi=\int_{\mathbb{R}^{d}}\left|w_{0}(\xi)\right| d \xi,
\end{aligned}
$$

which shows that $\Phi$ decreases along orbits if $w_{0}$ changes sign. The fact that $\Phi$ is constant when $w_{0}$ is everywhere non-negative or non-positive is easier and left as an exercise. 
Note that if we combine this Lemma with the LaSalle Invariance Principle we immediately have

Corollary 1.18 Any point in the $\omega$-limit set of a solution of (8) must be either everywhere positive, everywhere negative, or identically zero.

This Corollary may not seem very strong at first glance since one might think that all solutions just tend toward zero. However, this can be ruled out by the fact that solutions conserve the integral of the initial condition and conditions on the decay of solutions at infinity - thus, if the integral of the initial data is non-zero, we can conclude that the $\omega$-limit set is either everywhere positive or everywhere negative, a fact which will be important in the last lecture in this series. 


\section{Lecture 2: Invariant manifolds for partial differential equations on unbounded domains}

In this lecture we examine the application of invariant manifold theorems to some partial differential equations on unbounded spatial domains. For concreteness we focus primarily on the family of semi-linear heat equations:

$$
\begin{aligned}
\frac{\partial u}{\partial t} & =\Delta u-u|u|^{p-1}, \quad p>1 \\
u & =u(x, t), t \geq 0, \quad x \in \mathbb{R}^{d} .
\end{aligned}
$$

The long-time behavior of solutions of this equation have been intensively studied and not surprisingly the value of the exponent $p$ in the nonlinear term plays an essential role in this behavior. The dynamical systems approach described below gives a very simple explanation of this $p$-dependence.

Remark 2.19 There are a host of other applications of invariant manifold theorems to partial differential equations - see the references [1], [13] or [16] for a small sampling. We focus on this particular family of equations both because it will serve as a good "warm up" for treating the Navier-Stokes equations later and also because it illustrates one way of dealing with lack of a spectral gap which often arises in treating problems on unbounded spatial domains.

If one uses the Duhamel formula to convert (9) to an integral equation it is not difficult to show that this equation defines a smooth semigroup, at least for small initial data. However, if one tries to apply the invariant manifold theorem of Chen, Hale and Tan (CHT) described in the previous lecture one immediately runs into the problem that one cannot split the Banach space in the way described in hypotheses (H.3) and (H.4). The reason for this is a lack of a spectral gap and the origin of this problem is seen immediately even for the case of the linear heat equation

$$
\frac{\partial u}{\partial t}=\Delta u, \quad u=u(x, t), t \geq 0, x \in \mathbb{R}^{d} .
$$

For this equation we can immediately write down a representation of the semi-group. It is particularly easy to analyze in terms of the Fourier transform

$$
\hat{u}(k, t)=\frac{1}{(2 \pi)^{d / 2}} \int_{\mathbb{R}^{d}} u(x, t) e^{-i x \cdot k} d x
$$

If we are given initial conditions $u(x, 0)=u_{0} \in L^{2}\left(\mathbb{R}^{d}\right)$ then the solution of (10) can be written as:

$$
\hat{u}(k, t)=e^{-|k|^{2} t} \hat{u}_{0}(k)
$$

Since the semigroup in this case is just a multiplication operator we see that its spectrum equals the closed interval $[0,1]$. Since there is no gap in the spectrum there is no way to 
split the space of initial conditions in the way required by the (CHT) theorem. Thus, there is no easy way to identify subspaces of our Banach space which correspond to solutions with particular decay properties. A way to circumvent this problem emerges if one recalls the form of the fundamental solution of the heat equations:

$$
G(x, t)=\frac{1}{(4 \pi t)^{d / 2}} e^{-|x|^{2} /(4 t)} .
$$

Examining this solution we see that $x$ appears in a special way - namely as the combination $x / \sqrt{t}$ and this suggests that it might be more natural to study (10) not in terms of the independent variables $(x, t)$ but rather in terms of the new variable $\xi=x / \sqrt{t}$. With this in mind we introduce new dependent and independent variables through the definition:

$$
\begin{aligned}
& u(x, t)=\frac{1}{(1+t)^{\alpha / 2}} w\left(\frac{x}{\sqrt{1+t}}, \log (1+t)\right) \\
& \xi=\frac{x}{\sqrt{1+t}}, \quad \tau=\log (1+t)
\end{aligned}
$$

Note that in defining the new variables (often called "scaling" or "similarity" variables) we have defined $\xi=x / \sqrt{1+t}$ rather than $x / \sqrt{t}$ simply to avoid the singularity at $t=0$. This can be thought of as simply changing the origin of the time axis and since our equation is autonomous it has no effect on the problem. Also, the exponent $\alpha$ which occurs in the definition of $w$ will be chosen in a way convenient to each of the problems considered. For the moment, in our discussion of the linear heat equation we will take $\alpha=d$.

If we rewrite (10) in terms of these new variables we find that

$$
\begin{aligned}
& \frac{\partial w}{\partial \tau}=\mathcal{L} w, \quad w=w(\xi, \tau), \xi \in \mathbb{R}^{d} \\
& \mathcal{L} w=\Delta_{\xi} w+\frac{1}{2} \nabla \cdot(\xi w) .
\end{aligned}
$$

At first sight, this may not seem like an improvement as we have traded the heat equation for an apparently more complicated equation. However, as we will see this form of the equation has the advantage that a gap in the spectrum appears which separates the slowly decaying modes from the more rapidly decaying ones and allows us to apply the invariant manifold theorem of the preceeding lecture.

Remark 2.20 Note that (15) is precisely the equation considered in Example 1.16 at the end of the previous lecture. Since this equation is just the heat equation rewritten in new variables it is clear that solutions of this equation will inherit a maximum principle from the maximum principle satisfied by the heat equation.

To see why and how this spectral gap forms, consider the eigenvalue problem for $\mathcal{L}$ - for simplicity, we consider the case of $d=1$ though the following results are true in any dimension:

$$
\mathcal{L} \phi=\lambda \phi
$$


If we take the Fourier transform of this equation we find

$$
-|k|^{2} \hat{\phi}(k)-\frac{1}{2} k \frac{d \hat{\phi}}{d k}(k)=\lambda \phi(k)
$$

This first order equation can be solved with the aid of integrating factors and we find that for any $\lambda$ one has a solution

$$
\hat{\phi}^{\lambda}(k)=A^{+}|k|^{-2 \lambda} e^{-|k|^{2}} \Theta(k)+A^{-}|k|^{-2 \lambda} e^{-|k|^{2}} \Theta(-k),
$$

where $\Theta(k)$ is the Heaviside function. (Note that the singularity at the origin means we can have different constants $A^{+}$and $A^{-}$depending on whether $k$ is positive or negative.) Thus, we have a solution of the eigenvalue equation for any value of $\lambda$ so one might at first think that the spectrum of $\mathcal{L}$ is the whole complex plane. However, note that if $\lambda$ is real and positive, $\hat{\phi}^{\lambda}$ is singular at the origin and thus whether or not $\hat{\phi}^{\lambda}$ is an eigenfunction depends on what function space we are working on. This observation reminds us that in general the spectrum of an operator depends on its domain of definition and as we will see that is very true of the operator $\mathcal{L}$.

It has long been known that the time decay properties of parabolic equations are often connected with the spatial decay properties of their solutions. With this in mind we define the family of weighted Sobolev spaces:

$$
\begin{aligned}
L^{2}(m) & =\left\{f \in L^{2}\left(\mathbb{R}^{d}\right) \mid\|f\|_{m}<\infty\right\} \\
\|f\|_{m} & =\left(\int_{\mathbb{R}^{d}}\left(1+|\xi|^{2}\right)^{m}|f(\xi)|^{2} d \xi\right)^{1 / 2} \\
H^{s}(m) & =\left\{\partial^{\alpha} f \in L^{2}(m) \mid \text { for all } \alpha=\left(\alpha_{1}, \ldots, \alpha_{d}\right) \text { with }|\alpha| \leq s\right\}
\end{aligned}
$$

One standard property of these spaces which is very convenient for our subsequent use is that Fourier transformation is an isomorphism from $H^{s}(m)$ to $H^{m}(s)$. Thus, if we consider the spectrum of the operator $\mathcal{L}$ on the space $L^{2}(m)$, the point $\lambda$ will be in the spectrum if the function $\hat{\phi}^{\lambda} \in H^{m}(0)$ - i.e. if the function $\hat{\phi}^{\lambda}$ is in the "ordinary" Sobolev space $H^{m}$. Clearly $\hat{\phi}^{\lambda}$ is sufficiently smooth and rapidly decaying to be in $H^{m}$ for any $m$, provided we stay away from the origin. Thus, $\phi^{\lambda}$ will be in $H^{m}$ provided it, and all of its derivatives of order $m$ or less, are square integrable in some small neighborhood of the origin.

From the form of $\hat{\phi}^{\lambda}$ we see that the cases with $\lambda=-n / 2$ a non-positive half integer are "special". In this case, if we choose $A^{+}=(-1)^{n} A^{-}$we find that $\phi^{\lambda}(k)=c_{n} k^{n} e^{-|k|^{2}}$ is a solution of the eigenvalue equation. Since this function is entire and rapidly decaying $\hat{\phi}^{\lambda}$ is any Sobolev space $H^{m}$ and thus the points $\left\{-\frac{n}{2} \mid n=0,1,2 \ldots\right\}$ are in the spectrum of $\mathcal{L}$ when considered on any of the spaces $L^{2}(m)$. Furthermore, the corresponding eigenfunctions are given by the inverse Fourier transform of $k^{n} e^{-|k|^{2}}$ which implies $\phi^{n / 2}(\xi)=C_{n} \frac{d}{d \xi^{n}} e^{-\xi^{2} / 4}$. Of particular importance in our subsequent discussions will be the Gaussian eigenfunction of $\lambda=0, \phi^{0}(\xi)=\frac{1}{\sqrt{4 \pi}} e^{-\xi^{2} / 4}$, with the prefactor chosen so that $\phi^{0}$ has integral one. 
For other values of $\lambda$, the most singular behavior of $\hat{\phi}^{\lambda}$ and its derivatives will occur for the derivative of highest order and we see that near $k=0$ one has

$$
\frac{d^{m} \hat{\phi}^{\lambda}}{d k^{m}}(k) \sim|k|^{-2 \lambda-m} .
$$

This expression will be square integrable provided $2(2 \Re(\lambda)+m)<1$, i.e. if

$$
\Re(\lambda)<\frac{1}{4}-\frac{m}{2}
$$

Thus we have shown

Proposition 2.21 Fix $m>1$ and $d=1$ and let $\mathcal{L}$ be the operator in (15) acting on its maximal domain in $L^{2}(m)$. Then

$$
\sigma(\mathcal{L}) \supset\left\{\lambda \in \mathbb{C} \mid \operatorname{Re}(\lambda) \leq \frac{1}{4}-\frac{m}{2}\right\} \cup\left\{-\frac{n}{2} \mid n=0,1,2, \ldots\right\} .
$$

In addition to the eigenvalues computed in Proposition 2.21 the operator $\mathcal{L}$ could have continuous spectrum. However, it turns out that the spectrum in this case consists only of the eigenvalues already computed. Furthermore a similar calculation also works in higher dimensions and one finds:

Theorem 2.22 ([7], Theorem A.1) Fix $m>1$ and let $\mathcal{L}$ be the operator in (15) acting on its maximal domain in $L^{2}(m)$. Then

$$
\sigma(\mathcal{L})=\left\{\lambda \in \mathbb{C} \mid \operatorname{Re}(\lambda)<\frac{d}{4}-\frac{m}{2}\right\} \cup\left\{-\frac{n}{2} \mid n=0,1,2, \ldots\right\} .
$$

Although the spectral picture above gives valuable intuition about the behavior of the semigroup $e^{\tau \mathcal{L}}$, for later applications we will need more precise estimates on its properties. In particular, recall that the heat equation has strong smoothing properties (i.e. solutions of the heat equation with "rough" initial data are infinitely differentiable for all $t>0$ ) and we will need to know to what extent these smoothing properties survive when we introduce scaling variables.

Remark 2.23 Note that it is not automatic that the semigroup $e^{\tau \mathcal{L}}$ will be smoothing. From the spectral picture in Theorem 2.22 we see that the operator $\mathcal{L}$ is not sectorial in any of the $L^{2}(m)$ spaces. Thus in contrast to the heat equation semigroup, $e^{\tau \mathcal{L}}$ is not an analytic semigroup.

In addition to the smoothing properties of the semigroup for our later applications we will need to know what the spectral projection operators onto the various spectral subspaces of 
$\mathcal{L}$ are. From the discussion in Lecture we expect these to be given by eigenfuctions of the adjoint operator $\mathcal{L}^{\dagger}$. Formally the adjoint operator has the form

$$
\mathcal{L}^{\dagger} \psi=\Delta_{\xi} \psi-\frac{1}{2} \xi \cdot \nabla_{\xi} \psi
$$

If we specialize to one dimension again for simplicity the eigenvalue equation for $\mathcal{L}^{\dagger}$ is

$$
\mathcal{L}^{\dagger} \psi=\psi^{\prime \prime}-\frac{1}{2} \xi \psi^{\prime}=\lambda \psi
$$

This is Hermite's equation and thus, we find that the spectral projections are defined in terms of the Hermite polynomials. If $\alpha=\left(\alpha_{1}, \ldots, \alpha_{d}\right) \in \mathbb{N}^{d}$ we define

$$
H^{\alpha}(\xi)=\frac{2^{|\alpha|}}{\alpha !} e^{|\xi|^{2} / 4} \partial_{\xi}^{\alpha}\left(e^{-|\xi|^{2} / 4}\right)
$$

and then the projection $P_{n}$ onto the eigenspace corresponding to the eigenvalues $\lambda_{k}=$ $-\frac{k}{2}, k=0,1, \ldots, n$ is defined by

$$
\begin{aligned}
\left(P_{n} f\right)(\xi) & =\sum_{|\alpha| \leq n}\left(\int_{\mathbb{R}^{d}} H^{\alpha}\left(\xi^{\prime}\right) f\left(\xi^{\prime}\right) d \xi^{\prime}\right)^{1 / 2} \phi_{\alpha}(\xi) \\
\left(Q_{n} f\right)(\xi) & =\left(\left(\mathbf{1}-P_{n}\right) f\right)(\xi)
\end{aligned}
$$

We make two remarks about these projection operators that will be useful later.

Remark 2.24 The projection $P_{0}$ onto the zero eigenspace is simply

$$
\left(P_{0} f\right)(\xi)=\left(\int_{\mathbb{R}^{d}} f(z) d z\right) \phi^{0}(\xi)
$$

i.e. the projection of a function $f$ onto the zero eigenspace is just given by the product of the Gaussian, $\phi^{0}$, with the integral of $f$. In particular, any function of mean zero lies in the complementary subspace.

Remark 2.25 Following up on the preceding remark we see that a function $f$ lies in the range of $Q_{n}$ if and only if

$$
\int_{\mathbb{R}^{d}} \xi^{\alpha} f(\xi) d \xi=0
$$

for all $\alpha=\left(\alpha_{1}, \ldots, \alpha_{d}\right) \in \mathbb{N}^{d}$ with $|\alpha| \leq n$.

We now state our main technical estimate on the semigroup $e^{\tau \mathcal{L}}$. 
Proposition 2.26 Fix $n \in \mathbb{N} \cup\{-1\}$ and fix $m>n+1+\frac{d}{2}$ For all $\alpha \in \mathbb{N}^{d}$, there exists $C>0$ such that

$$
\left\|\partial^{\alpha}\left(e^{\tau \mathcal{L}} Q_{n} f\right)\right\|_{m} \leq \frac{C}{\left(1-e^{-\tau}\right)^{|\alpha| / 2}} e^{-\left(\frac{n+1}{2}\right) \tau}\|f\|_{m},
$$

for all $f \in L^{2}(m)$ and all $\tau>0$.

Proof: For the details of the proof we refer to [7], Appendix A. However, we note that the decay rate is exactly what we expect from the spectral picture in Theorem 2.22. The more delicate smoothing properties (quantified by the estimates of the derivatives of the semigroup) are obtained from the explicit integral representation of the semigroup which we easily obtain by noting that $e^{\tau \mathcal{L}} w_{0}$ is the solution of $(15)$ with the initial condition $w_{0}$ which when combined with (14) gives

$$
\left(e^{\tau \mathcal{L}} w_{0}\right)(\xi)=w(\xi, \tau)=e^{\frac{d}{2} \tau} u\left(\xi e^{\tau / 2}, e^{\tau}-1\right)
$$

and we then use the integral representation of $u$ in terms of $w_{0}$ which follows from the fact that $u$ solves the heat equation with initial condition $w_{0}$.

We now consider the implications of this result for the invariant manifold theorem. Recall that the problem with applying the invariant manifold theorem directly to the heat equation was that the semi-group had no spectral gap. If we now consider the semigroup defined by (15) then we see that the modes corresponding to the eigenvalues $\lambda=-\frac{n}{2}$ will decay like $e^{-\frac{n}{2} \tau}$ while modes lying in the half plane of essential spectra will all decay at least with a rate $e^{\left(\frac{d}{4}-\frac{m}{2}\right) \tau}$ and by choosing $m$ appropriately we can separate the decay rate of these modes from those associated with the first few eigenvalues. In particular, if we choose $m>d / 2$ we expect that as $t$ tends toward infinity solutions of (15) will approach a point on the eigenspace corresponding to the eigenvalue zero. Thus, we expect solutions of (15) to behave as

$$
w(\xi, t) \sim \frac{C_{0}}{(4 \pi)^{d / 2}} e^{-|\xi|^{2} / 4}
$$

as $t$ tends toward infinity, which just reflects, in these new variables, the fact that solutions of the heat equation tend toward a Gaussian profile as $t$ tends toward infinity. (Note that this is consistent with the conclusion of Example 1.16 where we showed that the $\omega$-limit set of non-zero solutions of (15) should be either everywhere positive or everywhere negative.)

We now turn to the nonlinear equation (9). We want to apply the results of (CHT) from the first lecture and to do that we need to study the semi-flow defined by this equation. We begin by rewriting the equation in terms of the scaling variables, (14). It is convenient if the resulting equation is autonomous and in order to insure that this is the case we pick the exponent $\alpha$ in the prefactor of $w$ to be $\alpha=\frac{2}{p-1}$. For what comes later it will be convenient to consider the exponent $p$ itself to be one of the dependent variables with a trivial time evolution. With this choice of exponent, and introducing an equation for $p,(9)$ is transformed into

$$
\begin{aligned}
\frac{\partial w}{\partial \tau} & =\mathcal{L} w+\left(\frac{1}{p-1}-\frac{d}{2}\right) w-|w|^{p-1} w \\
\frac{d p}{d \tau} & =0
\end{aligned}
$$


Here, $\mathcal{L}$ is exactly the same operator studied in connection with the heat equation and the change in the exponent $\alpha$ simply introduces the additional constant term $\left(\frac{1}{p-1}-\frac{d}{2}\right) w$ which just shifts the entire spectrum of $\mathcal{L}$ by that constant amount. Indeed, for simplicity in what follows we will focus particularly on the behavior of $p$ close to the value $\frac{d+2}{d}$ - i.e. close to the value for which this additional term vanishes. With this in mind, we exchange the variable $p$ for the variable $\eta$ defined by $p=1+\frac{2}{d+2 \eta}$ so that $\left(\frac{1}{p-1}-\frac{d}{2}\right) w=\eta w$, and recalling that $\eta$ (as was $p$ ) is considered to be one of the dependent variables this term can be considered a part of the nonlinearity! Thus, after these changes, we finally rewrite (9) in the form

$$
\begin{aligned}
& \frac{\partial w}{\partial \tau}=\mathcal{L} w+\eta w-|w|^{\frac{2}{d+2 \eta}} w \\
& \frac{d \eta}{d \tau}=0 .
\end{aligned}
$$

Now, to verify the hypotheses of the invariant manifold theorem of (CHT) we study the semiflow defined by this system of equations. The evolution of $\eta$ is trivial so we focus on the first component of the semiflow which we can write with the aid of Duhamel's formula as

$$
\Phi^{\tau}\left(w_{0}\right)=w(t)=e^{\tau \mathcal{L}} w_{0}+\int_{0}^{\tau} e^{(\tau-s) \mathcal{L}}\left(\eta w(s)-|w(s)|^{\frac{2}{d+2 \eta}} w(s)\right) d s,
$$

where we have suppressed the dependence of $w$ on $\xi$ to avoid overburdening the notation.

We now discuss the various hypotheses in the (CHT) theorem. The first is that $\Phi^{\tau}$ should be globally Lipshitz. This is not true of (35) due to the growth of the nonlinear term when $w$ becomes large. This is a standard problem with the application of invariant manifold theorems even in the context of ordinary differential equations and we handle it here in the same way it is usually handled in that setting, namely by "cutting off" the nonlinear term. Let $\chi(x)$ be a smooth, positive function on $\mathbb{R}$ satisfying

$$
\chi(x)= \begin{cases}1, & |x|<1 \\ 0, & |x|>2\end{cases}
$$

Then define

$$
\Phi_{r}^{\tau}\left(w_{0}\right)=w(t)=e^{\tau \mathcal{L}} w_{0}+\int_{0}^{\tau} e^{(\tau-s) \mathcal{L}}\left(\chi\left(\frac{\|w(s)\|_{m}}{r}\right)\left(\eta w(s)-|w(s)|^{\frac{2}{d+2 \eta}} w(s)\right)\right) d s,
$$

With this definition the nonlinear term vanishes if $\|w(s)\|_{m}$ is larger than $2 r$ but $\Phi_{r}^{\tau}\left(w_{0}\right)$ is equal to $\Phi^{\tau}\left(w_{0}\right)$ for all solutions that remain within a ball of radius $r$ in $L^{2}(m)$.

Remark 2.27 Note that the cutoff function $\chi\left(\frac{\|w(x)\|_{m}}{r}\right)$ is a smooth function on $L^{2}(m)$. It is always possible to find such a smooth cutoff function on a Hilbert space, but there are natural Banach spaces on which no such smooth cutoff function exists. This can cause problems for certain applications of invariant manifold theorems in infinite dimensional settings. 
It is now a standard exercise to verify that:

(N.1) $\Phi_{r}^{\tau}\left(w_{0}\right)$ is well defined for $w_{0} \in L^{2}(m)$.

(N.2) The nonlinear term

$$
\mathcal{R}_{r}^{\tau}\left(\eta, w_{0}\right)=\int_{0}^{\tau} e^{(\tau-s) \mathcal{L}}\left(\chi\left(\frac{\|w(x)\|_{m}}{r}\right)\left(\eta w(s)-|w(s)|^{\frac{2}{d+2 \eta}} w(s)\right)\right) d s
$$

is globally Lipshitz with Lipshitz constant bounded by $C_{\mathcal{R}}\left(\eta+r^{\frac{2}{d+2 \eta}}\right)$ for some constant $C_{\mathcal{R}}$. Thus, the Lipshitz constant can be made arbitrarily small for $\eta$ and $r$ sufficiently small.

These two observations are sufficient to verify hypotheses (H.1) and (H.2) of the of the (CHT) theorem. (We can choose the constants $q=\tau=1$ and set $\Lambda=e^{\tau \mathcal{L}}$ and $\mathcal{R}=\mathcal{R}_{r}^{1}$.)

We next verify hypothesis (H.3). Here we must make a choice. Given any $n=0,1,2, \ldots$ we could, by choosing $m$ appropriately, set $X_{1}=P_{n} L^{2}(m)$ and $X_{2}=Q_{n} L^{2}(m)$. We would then obtain an invariant manifold tangent at the origin to the eigenspace corresponding to the eigenvalues $\left\{-\frac{k}{2} \mid k=0,1, \ldots, n\right\}$. The long-time behavior of solutions close to the origin could then be determined up to corrections which go to zero at least as fast as $e^{-\gamma \tau}$ with $\gamma>n / 2$ just by studying the asymptotics of solutions of the finite dimensional system of ordinary differential equations which results from restricting (34) to this invariant manifold.

For now we focus on the simplest possible case, namely we will assume that $m>\max (1, d / 2)$ and take $X_{1}=P_{0} L^{2}(m)$. In this case $X_{1}$ is the one dimensional subspace spanned by $\phi^{0}(\xi)=\frac{1}{(4 \pi)^{d / 2}} e^{-|\xi|^{2} / 4}$. Next we find

$$
\Lambda_{1}=P_{0} e^{\tau \mathcal{L}} P_{0}=\mathbf{1},
$$

the identity operator and we can take the constants $C_{1}=\alpha_{1}=1$ in hypothesis (H.3). Then

$$
\Lambda_{2}=Q_{0} e^{\tau \mathcal{L}} Q_{0}
$$

and from Proposition 2.26 we see that (H.3) holds for $\alpha_{2}=e^{-\left(\frac{m}{2}-\frac{d}{2}\right)}<1$ and for some $C_{2}>0$.

Finally, condition (H.4) is satisfied since by remark (N.2) the Lipshitz constant of $\mathcal{R}$ can be made arbitrarily small for $\eta$ and $r$ sufficiently small.

Since we are considering $\eta$ to be one of the dependent variables we should also consider the evolution of $\eta$ - however, this evolution is trivial and hence we can just apply the (CHT) theorem to $\Phi^{\tau=1}$ for each value of $\eta$ small, treating $\eta$ as a parameter.

Applying the (CHT) theorem we conclude 
Proposition 2.28 Fix $m>\max (1, d / 2)$. There exists $r_{0}>0$ and $\eta_{0}>1$ such that if $|\eta|<\eta_{0}$ and $0<r<r_{0}$ there exists a globally Lipshitz map $g: P_{0} L^{2}(m) \rightarrow Q_{0} L^{2}(m)$ with $g(0)=D g(0)=0$ such that the submanifold

$$
W_{c}=\left\{\alpha \phi^{0}+g\left(\alpha \phi^{0}\right) \mid \alpha \in \mathbb{R}\right\}
$$

has the following properties:

(i) (Invariance) $\Phi^{\tau}$ leaves $W_{c}$ invariant.

(ii) (Fixed Points) If $\{w(t)\}_{\tau \leq 0}$ is a negative semi-orbit with $\|w(\tau)\|_{m} \leq r_{0}$ for all $\tau \leq 0$, then $w(\tau) \in W_{c}$ for all $\tau$.

(iii) (Attractivity) Fix $\mu$ such that $0<\mu<\min \left(\frac{1}{2}, \frac{d}{4}-\frac{m}{2}\right)$. There exists $C$ and $r_{2}$, positive constants, such that for $\tilde{w}_{0} \in L^{2}(m)$ with $\left\|\tilde{w}_{0}\right\|_{m}<r_{2}$, there exists a unique $w_{0} \in W_{c}$ such that

$$
\left\|\Phi^{\tau}\left(\tilde{w}_{0}\right)-\Phi^{\tau}\left(w_{0}\right)\right\|_{m} \leq C e^{-\mu \tau} .
$$

Remark 2.29 The "Fixed Points" and "Attractivity" parts of the conclusions of this theorem follow respectively from the "Lyapunov Exponents" and "Invariant Foliation" parts of the (CHT) theorem if we use the fact that in this problem we can choose $\gamma_{2}<1$. In particular, $\mu=-\log \gamma_{2}$.

Note that since, for $\|w(\tau)\|_{m}<r$ the semiflow $\Phi_{r}^{\tau}$ coincides with $\Phi^{\tau}$, the semiflow for (34) the rescaled heat equation will also have a local invariant manifold which attracts all solutions in some sufficiently small neighborhood of the origin.

We conclude this lecture by considering the implications of this manifold for the long-time behavior of solutions of (34). From the "Attractivity" part of Proposition 2.28, the long-time behavior of small solutions of (34) will (up to higher order corrections) be the same as those of solutions lying on the manifold $W_{c}$, and the long-time behavior of solutions lying on this manifold can be determined by solving the single ordinary differential equation that results from restricting the original partial differential equation to this manifold. If $w(\xi, \tau)$ lies on $W_{c}$ we can write

$$
w(\xi, \tau)=\alpha(\tau) \phi^{0}(\xi)+g\left(\alpha(\tau) \phi^{0}(\xi)\right) .
$$

Inserting this representation of $w$ into (34) gives

$$
\begin{aligned}
\dot{\alpha}(\tau) \phi^{0}(\xi)+\dot{\alpha}(\tau) D g\left(\alpha(\tau) \phi^{0}(\xi)\right) \phi^{0}(\xi)= \\
=\alpha(\tau)\left(\mathcal{L} \phi^{0}\right)(\xi)+\eta \phi^{0}(\xi)+\mathcal{L} g\left(\alpha(\tau) \phi^{0}(\xi)\right)+\eta g\left(\alpha(\tau) \phi^{0}(\xi)\right) \\
\quad-\left|\alpha(\tau) \phi^{0}(\xi)+g\left(\alpha(\tau) \phi^{0}(\xi)\right)\right|^{\frac{2}{d+2 \eta}}\left(\alpha(\tau) \phi^{0}(\xi)+g\left(\alpha(\tau) \phi^{0}(\xi)\right)\right)
\end{aligned}
$$

We now reduce this to an ordinary differential equation for $\alpha(\tau)$ by noting that $\mathcal{L} \phi^{0}=0$ and then applying the projection operator $P_{0}$ to both sides of the equation. This yields:

$$
\dot{\alpha}(\tau)=\eta \alpha(\tau)-\int_{\mathbb{R}}\left|\alpha(\tau) \phi^{0}(\xi)+g\left(\alpha(\tau) \phi^{0}(\xi)\right)\right|^{\frac{2}{d+2 \eta}}\left(\alpha(\tau) \phi^{0}(\xi)+g\left(\alpha(\tau) \phi^{0}(\xi)\right)\right) d \xi
$$


For the moment the only thing we need to know about the complicated nonlinear term is that since $g(0)=0$, and $D g(0)=\mathcal{O}(\eta)$ (by explicit computation of the equation satisfied by the invariant manifold), for $\alpha$ and $\eta$ small it behaves like $C_{L}|\alpha|^{\frac{2}{d+2 \eta}} \alpha$, where $C_{L}=\int_{\mathbb{R}}\left(\phi^{0}(\xi)\right)^{p} d \xi+$ $\mathcal{O}(\eta)$

From this equation is clear that varying $\eta$ (or equivalently $p$ ) leads to a bifurcation at $\eta=0$. From now on, for simplicity we assume that $d=1$, though the computations can be carried through in a similar way for higher dimensions. Note that in $d=1, \eta=0$ corresponds to the exponent $p=3$. To better understand the bifurcation that results when we vary $\eta$ we first consider solutions of (43) when $\eta<0$ which corresponds to $p>3$. In this case the origin is an attractive fixed point for (43) and for any solutions with $\alpha(0)$ sufficiently small we have

$$
\alpha(\tau) \sim C_{0} e^{-\eta \tau}
$$

for some $C_{0}>0$. From this we immediately conclude that solutions on the invariant manifold $W_{c}$ behave for large times like

$$
\begin{aligned}
w(\xi, \tau) & =C_{0} e^{-\eta \tau} \phi^{0}(\xi)+g\left(C_{0} e^{-\eta \tau} \phi^{0}(\xi)\right) \\
& =C_{0} e^{-\eta \tau} \phi^{0}(\xi)+\mathcal{O}\left(e^{-2 \eta \tau}\right)
\end{aligned}
$$

where the last equality reflects the fact that since $g(0)=D g(0)=0$ (which follows from a direct inspection of the equation satisfied by the function $g$.) Furthermore by the "Attractivity" part of Proposition 2.28 all small solutions will behave like (44) to leading order. Thus we have:

Corollary 2.30 All sufficiently small solutions of (34), behave asymptotically like

$$
w(\xi, \tau)=C_{0} e^{-\eta \tau} \phi^{0}(\xi)+\mathcal{O}\left(e^{-2 \eta \tau}\right)
$$

for some constant $C_{0}$.

Note that from this corollary it appears that the decay rate of these solutions depends on $p$ through the exponent $\eta$. However, if we revert to our original variables we see that solutions of the original equation (9) behave as

$$
\begin{aligned}
u(x, t) & =\frac{1}{(1+t)^{\alpha / 2}} w\left(\frac{x}{\sqrt{1+t}}, \log (1+t)\right)=\frac{1}{(1+t)^{\frac{1}{(p-1)}}} w\left(\frac{x}{\sqrt{1+t}}, \log (1+t)\right) \\
& =\frac{1}{(1+t)^{\frac{1}{(p-1)}}}\left(C_{0}(1+t)^{-\eta} \phi^{0}\left(\frac{x}{\sqrt{1+t}}\right)+\mathcal{O}\left((1+t)^{-2 \eta}\right)\right. \\
& =\frac{C_{0}}{\sqrt{1+t}} \phi^{0}\left(\frac{x}{\sqrt{1+t}}\right)+\ldots
\end{aligned}
$$

Thus, for $p>3$, all small solutions of (9) behave to leading order as if the nonlinear term was absent - such nonlinear terms are often referred to as "irrelevant". 
Let's now consider what happens if $p<3$ (or $\eta>0$ ). In this case the origin is unstable and the fixed point at the origin undergoes a pitchfork bifurcation and a pair of new fixed points appears at $\pm \alpha^{*} \approx \pm\left(\eta / C_{L}\right)^{\frac{1}{p-1}}$. These fixed points are stable (at least for $\eta$ sufficiently small) and hence all non-zero solutions in $W_{c}$ will approach one of them. Define

$$
w^{*}(\xi ; p)=\alpha^{*} \phi^{0}(\xi)+g\left(\alpha^{*} \phi^{0}(\xi)\right) .
$$

Then, small solutions of (9) will behave like

$$
w(\xi, \tau) \approx w^{*}(\xi)
$$

for $\tau$ large.

Remark 2.31 In fact, there are some solutions which will approach the origin even when $\eta<0$. Those are the solutions that lie in the stable manifold of the origin. However, these solutions for a manifold of codimension-one and hence "most" solutions will behave as in (46).

If we again revert to the original variables we find

Corollary 2.32 For $p<3$, all sufficiently small solutions of (9) except for those lying in the codimension one stable manifold of the origin, behave like

$$
u(x, t)=\frac{1}{(1+t)^{\frac{1}{p-1}}} w^{*}\left(\frac{x}{\sqrt{1+t}}\right)+\ldots .
$$

Thus, we see that for $p<3$ the situation is quite different from that for $p>3$ since both the rate of decay of the long-time asymptotics and the functional form of the limiting solution depend on the nonlinear term.

Exercise 2.33 Determine the behavior of the long-time asymptotics of solutions when $p=3$ - the "critical" value of the nonlinear term.

Remark 2.34 By considering the manifolds tangent to the spectral subspaces corresponding to more than just the zero eigenvalue - say to the eigenvalues $\left\{0,-\frac{1}{2}\right\}$ or $\left\{0,-\frac{1}{2},-1\right\}$, etc. one can derive more refined estimates of the long-time behavior of the solutions.

Summing up this lecture, we have found a way, at least in some parabolic partial differential equations, to create a spectral gap which allows us to apply invariant manifold theorems to problems on unbounded spatial domains. These theorems can then give detailed information about the long-time asymptotics of solutions. The drawback is that these results are local in nature - in the present examples they apply only to "small" solutions. As we'll see in the fourth lecture in this series that restriction can sometimes be lifted by combining these results with Lyapunov functionals which give more global control over the solutions. 


\section{Lecture 3: An introduction to the Navier-Stokes equa- tions}

In this section we will discuss the Navier-Stokes equations which describe the velocity of a viscous, incompressible fluid. The focus of this lecture will be the origin of the equations, their representation in terms of both the velocity and vorticity of the fluid and the existence of solutions in the two dimensional case. In the final lecture in this series we will look in greater detail at the long-time behavior of solutions of the two-dimensional Navier-Stokes equations. A more detailed look at the Navier-Stokes equation, but with a similar point of view can be found in the lecture notes of Gallagher and Gallay [6]. For more discussion of the physical origin of these equations one can consult [5].

The Navier-Stokes equations arise from applying Newton's law to determine the motion of a small "blob" of fluid. Assume that the "blob" is a cube of side length $\Delta x$, centered at the point $x \in \mathbb{R}^{d}$, where for physical relevance we restrict to the cases $d=2$ or 3 . Newton's Law implies

$$
\frac{d}{d t}(\text { momentum })=\text { applied forces . }
$$

If $\mathbf{u}(x, t)$ is the fluid's velocity measured in the laboratory frame of reference, and if the density of the fluid is $\rho$, then the momentum will be $\boldsymbol{\pi}(x, t)=\rho(x, t) \mathbf{u}(x, t) \Delta V$, where $\Delta V$ is the volume of the little cube of fluid. To simplify the discussion we will assume that the density $\rho$ is constant and check a posteriori that this is consistent with the equations of motion. We'll also ignore the factor of $\Delta V$ since it will occur in each term and can be cancelled out.

To compute the change in moment of our fluid blob we need to take account of the fact that the fluid is being advected along by its own velocity. Thus,

$$
\begin{aligned}
\frac{d \boldsymbol{\pi}}{d t}(x, t) & =\lim _{\Delta t \rightarrow 0} \frac{\boldsymbol{\pi}(x+\mathbf{u}(x, t) \Delta t, t+\Delta t)-\boldsymbol{\pi}(x, t)}{\Delta t} \\
& =\mathbf{u}(x, t) \cdot \nabla \boldsymbol{\pi}(x, t)+\frac{\partial \boldsymbol{\pi}}{\partial t}(x, t) .
\end{aligned}
$$

This expression is known as the convective derivative of the momentum. Thus, returning to Newton's law, we have

$$
\frac{\partial \boldsymbol{\pi}}{\partial t}(x, t)+\mathbf{u}(x, t) \cdot \nabla \boldsymbol{\pi}(x, t)=\text { applied forces } .
$$

What are the forces that act on the fluid element?

- forces due to pressure: $f_{\text {pressure }}=-\nabla p(x, t)$, where $p$ is the pressure in the fluid.

- external forces: we'll ignore these.

- viscous forces: These involve modeling internal properties of the fluid. We will take a standard model which says $f_{\text {visc }}=\alpha \Delta \mathbf{u}$, for some constant $\alpha$. 
Inserting these forces into Newton's law we arrive at the system of partial differential equations:

$$
\frac{\partial \boldsymbol{\pi}}{\partial t}(x, t)+\mathbf{u}(x, t) \cdot \nabla \boldsymbol{\pi}(x, t)=\alpha \Delta \mathbf{u}(x, t)-\nabla p(x, t)
$$

Assuming that the density is constant, this is a system of $d$ equations, but it contains $d+1$ unknowns - the $d$ components of the velocity, plus the pressure. We need one further equation linking the pressure and momentum in order to close the system. This remaining equation is derived from the property of conservation of mass. If we look at the equation for the change in the amount of mass in a region $V$, then we see that by conservation of mass, any change in the mass in the region (given by $\int_{V}\left(\partial_{t} \rho\right) d V$ ) must be counterbalanced by a flux of mass through the boundary (given by $-\int_{\partial V} \rho \mathbf{u} \cdot \hat{\mathbf{n}} d S$ ). Equating these two expressions, applying the divergence theorem and using the fact that the region $V$ was arbitrary leads to the conservation equation

$$
\frac{\partial \rho}{\partial t}(x, t)+\nabla \cdot(\mathbf{u}(x, t) \rho(x, t))=0
$$

If we now impose the incompressibility condition $\nabla \cdot(\mathbf{u}(x, t) \rho(x, t))=0$ we see that $\rho(x, t)=$ $\rho(x, 0)$. In particular, if the density is initially constant it will remain so for all time and the incompressibility condition simplifies to $\nabla \cdot(\mathbf{u}(x, t))=0$. Then we have a system of $d+1$ nonlinear partial differential equations:

$$
\begin{aligned}
\rho \frac{\partial \mathbf{u}}{\partial t}(x, t)+\rho \mathbf{u}(x, t) \cdot \nabla \mathbf{u}(x, t) & =\alpha \Delta \mathbf{u}(x, t)-\nabla p(x, t) \\
\nabla \cdot \mathbf{u}(x, t) & =0 .
\end{aligned}
$$

Remark 3.35 Note that given a solution $\mathbf{u}$ of (48) one can recover the pressure by taking the divergence of the momentum equation and using the incompressibility equation from which one finds:

$$
\Delta p=-\rho \nabla \cdot(\mathbf{u} \cdot \nabla \mathbf{u}),
$$

so the pressure is obtained as a solution of Poisson's equation.

Remark 3.36 The coefficients in (48) can be simplified somewhat. Suppose that we introduce some fixed length scale $L$, velocity scale $V$ and reference density $\bar{\rho}$. If we define new, dimensionless variables via $\tilde{x}=x / L, \tilde{\mathbf{u}}=\mathbf{u} / V, \tilde{t}=(t V) / L$, and $\tilde{\rho}=\rho / \bar{\rho}$, then a simple exercise shows that in terms of the new variables (48) is replaced by:

$$
\frac{\partial \tilde{\mathbf{u}}}{\partial \tilde{t}}+\tilde{\mathbf{u}} \cdot \nabla \tilde{\mathbf{u}}=\tilde{\alpha} \Delta \tilde{\mathbf{u}}-\frac{1}{\tilde{\rho}} \nabla \tilde{p}
$$

where $\tilde{\alpha}=\frac{\alpha}{\rho V^{2} L}, \tilde{p}=p /(\bar{\rho} L)$, and all derivatives are computed with respect to the new variables. These changes of variables are particularly convenient if we study this equation on the domain $\mathbb{R}^{d}$ since in this case the rescaling has no effect on the domain and if we choose the length scale $L=\alpha /\left(\rho V^{2}\right)$, all coefficients in the equation become equal to one. From now on we will assume that we have made these changes of variables and drop the tildes to avoid burdening the notation. 
Remark 3.37 A related quantity is the dimensionless ratio of the inertial forces to the viscous forces given by

$$
\operatorname{Re}=\frac{\left(\rho V^{2} / L\right)}{\left(\alpha V / L^{2}\right)}=\frac{\rho V L}{\alpha}
$$

known as the Reynolds number.

The remainder of this lecture will be devoted to studying the initial value problem for (48) - namely given some initial velocity distribution $\mathbf{u}(x, 0)=\mathbf{u}_{0}(x)$, show that the equation (48) has a unique solution and describe the properties of this solution. Proving that (48) has a unique, smooth solution for all initial data is a very famous problem. Basically, two alternatives have developed so far:

- give up smoothness and uniqueness and simply try to show that there is some (weak) solution to the problem. This approach dates back to the work of Leray.

- attempt to show that the initial value problem is well posed, at the expense of specializing the problem somehow - perhaps considering "small" initial data, or restricting the domain on which the problem is posed.

I'll adopt the second approach in these lectures by focusing on the two-dimensional problem. When studying the two-dimensional Navier-Stokes equation defined in the entire plane it turns out to be simpler to work not directly with the velocity field but rather with the vorticity of the fluid. The vorticity is defined by the curl of the velocity field - i.e. $\boldsymbol{\omega}(x, t)=$ $\nabla \times \mathbf{u}(x, t)$ and in general it is a vector field, just like the velocity. However, in two dimensions

$$
\boldsymbol{\omega}(x, t)=\nabla \times\left(u_{1}\left(x_{1}, x_{2}, 0, t\right), u_{2}\left(x_{1}, x_{2}, 0, t\right), 0\right)=\left(0,0, \omega\left(x_{1}, x_{2}, t\right)\right)
$$

so we see that only one component of the vorticity is non-zero and thus we may treat it as a scalar. If we take the curl of the Navier-Stokes equation we find that (in general dimension d)

$$
\frac{\partial \boldsymbol{\omega}}{\partial t}+\boldsymbol{\omega} \cdot \nabla \mathbf{u}+\mathbf{u} \cdot \nabla \boldsymbol{\omega}=\Delta \omega
$$

Note that another advantage of the vorticity formulation of the problem is that the pressure term drops out entirely.

Remark 3.38 The term $\boldsymbol{\omega} \cdot \nabla \mathbf{u}$ is known as the "vorticity stretching term". It allows for a certain "self amplification" of the vorticity. Note that in two dimensions this term is zero since $\boldsymbol{\omega} \cdot \nabla \mathbf{u}=\omega \partial_{x_{3}} \mathbf{u}\left(x_{1}, x_{2}, 0\right)=0$. The absence of this term is another reason why the two-dimensional Navier-Stokes (or vorticity) equation is easier to treat than the three dimensional one. 
From now on we will restrict our attention to the two-dimensional vorticity equation and consider the initial value problem

$$
\begin{aligned}
& \frac{\partial \omega}{\partial t}+\mathbf{u} \cdot \nabla \omega=\Delta \omega, t>0, x \in \mathbb{R}^{2} \\
& \omega(x, 0)=\omega_{0}(x)
\end{aligned}
$$

The principal difficulty in studying (50) is the presence of the velocity, $\mathbf{u}$, in this equation. We must reconstruct the velocity from the vorticity - however, this leads to a somewhat complicated, nonlocal nonlinearity. Recalling that the vorticity is the curl of the velocity and that the velocity is incompressible, we can reconstruct the velocity using the Biot-Savart law

$$
\mathbf{u}(x)=\frac{1}{2 \pi} \int_{\mathbb{R}^{2}} \frac{(x-y)^{\perp}}{|x-y|^{2}} \omega(y, t) d y .
$$

Here, for any two dimensional vector $x=\left(x_{1}, x_{2}\right)$ we define $x^{\perp}=\left(-x_{2}, x_{1}\right)$.

Exercise 3.39 Verify that the Biot-Savart law does give an incompressible velocity field whose curl is the vorticity.

In order to control the solutions of (50) (and to verify the hypotheses of the (CHT) theorem) we need estimates which relate the norm of the velocity to the vorticity. A collection of such estimates is derived in [7], Appendix B, but as an example of the sort of estimates one needs we prove:

Lemma 3.40 Let $\mathbf{u}$ be the velocity field associated to the vorticity $\omega$ by the Biot-Savart law. Fix $1<q<2$. Then if

$$
\frac{1}{q}-\frac{1}{p}=\frac{1}{2}
$$

there exists $C=C(p, q)$ such that

$$
\|\mathbf{u}\|_{L^{p}\left(\mathbb{R}^{2}\right)} \leq C\|\omega\|_{L^{q}\left(\mathbb{R}^{2}\right)}
$$

Remark 3.41 Define the $L^{p}$ norm of a vector valued function as the sum of the $L^{p}$ norms of the components.

Proof: Recall the Hardy-Littlewood-Sobolev Inequality

$$
\int_{\mathbb{R}^{d}} f(x)\left(\int_{\mathbb{R}^{d}} \frac{1}{|x-y|^{\lambda}} g(y) d y\right) d x \leq N(p, q, \lambda, d)\|f\|_{L^{s}\left(\mathbb{R}^{d}\right)}\|g\|_{L^{q}\left(\mathbb{R}^{d}\right)},
$$

provided $\frac{1}{s}+\frac{1}{q}+\frac{\lambda}{d}=2$. Note that

$$
\left|u_{j}(x, t)\right| \leq \frac{1}{2 \pi} \int_{\mathbb{R}^{2}} \frac{1}{|x-y|}|\omega(y, t)| d y \equiv h(x)
$$


so that $\left\|u_{j}(x, t)\right\|_{L^{p}\left(\mathbb{R}^{2}\right)} \leq\|h\|_{L^{p}\left(\mathbb{R}^{2}\right)}$. Let $f=h^{p-1}$. Then applying the HLS inequality we find

$$
\|h\|_{L^{p}\left(\mathbb{R}^{2}\right)}^{p} \leq N\left\|h^{p-1}\right\|_{L^{s}\left(\mathbb{R}^{2}\right)}\|\omega\|_{L^{q}\left(\mathbb{R}^{2}\right)},
$$

Take $s=\frac{p}{p-1}$. Then $\left\|h^{p-1}\right\|_{L^{s}\left(\mathbb{R}^{2}\right)}=\|h\|_{L^{p}\left(\mathbb{R}^{2}\right)}^{p-1}$ and hence

$$
\|h\|_{L^{p}\left(\mathbb{R}^{2}\right)} \leq N\|\omega\|_{L^{q}\left(\mathbb{R}^{2}\right)},
$$

with $\frac{1}{q}-\frac{1}{p}=\frac{1}{2}$

Exercise 3.42 Use the Biot-Savart law to prove that

$$
\|\mathbf{u}\|_{L^{\infty}\left(\mathbb{R}^{2}\right)} \leq C\left(\|\omega\|_{L^{1}\left(\mathbb{R}^{2}\right)}+\|\omega\|_{L^{\infty}\left(\mathbb{R}^{2}\right)}\right) .
$$

We now have the tools we need to prove the existence and uniqueness of solutions of the twodimensional vorticity equation. This is a story with a long history but the approach I describe below was first developed by Ben-Artzi, [2]. My presentation of this approach is close to that of [6]. The first question that arises it what space we should work in. Note that if $\omega(x, t)$ solves $(50)$, then so does $\omega_{\lambda}(x, t)=\lambda^{2} \omega\left(\lambda x, \lambda^{2} t\right)$ for any $\lambda$. Since $\left\|\omega_{\lambda}(\cdot)\right\|_{L^{1}\left(\mathbb{R}^{2}\right)}=\|\omega(\cdot)\|_{L^{1}\left(\mathbb{R}^{2}\right)}$, this suggests that the space $L^{1}\left(\mathbb{R}^{2}\right)$ is appropriate and it turns out that in this space all initial conditions lead to unique global solutions. More precisely one has

Theorem 3.43 There exists $C>0$ such that for any $\omega_{0} \in L^{1}\left(\mathbb{R}^{2}\right)$, the initial value problem (50) has a unique solution $u \in C\left(\mathbb{R}^{+} ; L^{1}\left(\mathbb{R}^{2}\right)\right)$.

Proof: The proof basically consists of two steps:

(1.) One first shows that given $\omega_{0} \in L^{1}\left(\mathbb{R}^{2}\right)$, the initial value problem has a unique solution for some interval of time $T_{0}$. Furthermore, for any positive time this solution is in $L^{p}\left(\mathbb{R}^{2}\right)$ for all $1 \leq p \leq \infty$.

(2.) Show that if the initial condition $\omega_{0} \in L^{1}\left(\mathbb{R}^{2}\right) \cap L^{\infty}\left(\mathbb{R}^{2}\right)$ then one has a unique solution for all time.

Note that these points taken together suffice to prove the theorem since given an initial condition $\omega_{0} \in L^{1}\left(\mathbb{R}^{2}\right)$ we first solve the initial value problem for some short time. We then take this solution at some positive time $t_{0}$ as our new initial condition and the resulting solution exists for all time.

I'll look in detail at the second part of the proof - details of the first part can be found in [2] or [6]. As a first step we rewrite (50) as an integral equation, just as we did with the semi-linear heat equation in Lecture 2.

$$
\omega(t)=\Phi^{t}\left(\omega_{0}\right)=e^{t \Delta} \omega_{0}+\int_{0}^{t} e^{(t-s) \Delta} \mathbf{u}(s) \cdot \nabla \omega(s) d s,
$$


where $e^{t \Delta}$ denotes the semigroup defined by the heat equation. The proof of the theorem now follows by showing that (52) has a fixed point in an appropriate Banach space.

Remark 3.44 Before beginning the fixed point argument, however, we note that if (50) has a solution, the solution has the following important property. In two dimensions, since the vorticity is a scalar, it satisfies the maximum principle. As a consequence not only is the $L^{1}\left(\mathbb{R}^{2}\right)$ norm a non-increasing function of time (remember Example 1.16) but in fact by a similar argument one finds that $\|\omega(t)\|_{L^{p}\left(\mathbb{R}^{2}\right)} \leq\left\|\omega_{0}\right\|_{L^{p}\left(\mathbb{R}^{2}\right)}$ for all $1 \leq p \leq \infty$.

Returning to (52) we write this equation as

$$
\omega(t)=\mathcal{F}(\omega)(t)=e^{t \Delta} w_{0}+\mathcal{N}(w, w)(t)
$$

where

$$
\mathcal{N}(\tilde{\omega}, \omega)(t)=\int_{0}^{t} e^{(t-s) \Delta} \tilde{\mathbf{u}}(s) \cdot \nabla \omega(s) d s,
$$

and $\tilde{\mathbf{u}}$ is the velocity field associated to the vorticity $\tilde{\omega}$ by the Biot-Savart law. Note that $\tilde{\mathbf{u}}$ is a linear function of $\tilde{\omega}$ so $\mathcal{N}$ is a bilinear operator. We'll study the fixed point problem for $\mathcal{F}$ on the Banach space

$$
X_{T}^{*}=\left\{f \in C\left([0, T]: L^{1}\left(\mathbb{R}^{2}\right) \cap L^{\infty}\left(\mathbb{R}^{2}\right)\right)\right\}
$$

with norm $\|f\|_{*}=\sup _{0 \leq t \leq T}\left(\|f(t)\|_{L^{1}\left(\mathbb{R}^{2}\right)}+\|f(t)\|_{L^{\infty}\left(\mathbb{R}^{2}\right)}\right)$.

We first note that if $\omega_{0} \in L^{1}\left(\mathbb{R}^{2}\right) \cap L^{\infty}\left(\mathbb{R}^{2}\right)$ then the linear term in (53) is an element of $X_{T}^{*}$. This follows immediately from the estimates:

Lemma 3.45 For any $\alpha=\left(\alpha_{1}, \alpha_{2}\right) \in \mathbb{N}^{2}$ and $1 \leq p \leq q \leq \infty$ there exists $C=C(p, q, \alpha)$ such that

$$
\left\|\partial^{\alpha}\left(e^{t \Delta} f\right)\right\|_{L^{p}\left(\mathbb{R}^{2}\right)} \leq \frac{C}{t^{\frac{|\alpha|}{2}+\left(\frac{1}{q}-\frac{1}{p}\right)}}\|f\|_{L^{q}\left(\mathbb{R}^{2}\right)} .
$$

Proof: The proof of this lemma follows easily by applying Young's inequality to the explicit integral representation for the heat semigroup.

The key estimate is the following bound on the nonlinear term:

Lemma 3.46 There exists $C>0$ such that for any $\tilde{\omega}$ and $\omega$ in $X_{T}^{*}$,

$$
\|\mathcal{N}(\tilde{\omega}, \omega)\|_{*} \leq C \sqrt{T}\|\tilde{\omega}\|_{*}\|\omega\|_{*} .
$$


Assuming for the moment that the lemma holds we proceed as follows. Given the estimates of the two preceeding lemmas a standard application of the contraction mapping theorem shows that (53) has a unique fixed point in $X_{T}^{*}$ provided

$$
4 C \sqrt{T}\|\omega\|_{*} \leq 1 .
$$

However, this estimate is problematic since it involves the fixed point itself and hence makes it difficult to get a good estimate of the time of existence of the solution (which we want ultimately to show is infinity.) We now make use of Remark 3.44. from which we conclude that $\|\omega\|_{*} \leq\left(\left\|\omega_{0}\right\|_{L^{1}\left(\mathbb{R}^{2}\right)}+\left\|\omega_{0}\right\|_{L^{\infty}\left(\mathbb{R}^{2}\right)}\right)$. But if we couple this observation with (55) we see that we obtain a unique solution of (50) for all times $0 \leq t \leq T$ such that

$$
T=\left(\frac{1}{4 C\left(\left\|\omega_{0}\right\|_{L^{1}\left(\mathbb{R}^{2}\right)}+\left\|\omega_{0}\right\|_{L^{\infty}\left(\mathbb{R}^{2}\right)}\right)}\right)^{2} .
$$

In order to show that this solution actually exists for all time we now repeat this procedure, taking as our new initial condition $\tilde{\omega}_{0}=\omega(T)$. This new solution (which is the continuation of our original solution) exists for at least a time

$$
\tilde{T}=\left(\frac{1}{4 C\left(\left\|\tilde{\omega}_{0}\right\|_{L^{1}\left(\mathbb{R}^{2}\right)}+\left\|\tilde{\omega}_{0}\right\|_{L^{\infty}\left(\mathbb{R}^{2}\right)}\right)}\right)^{2}
$$

However, since

$$
\left(\left\|\tilde{\omega}_{0}\right\|_{L^{1}\left(\mathbb{R}^{2}\right)}+\left\|\tilde{\omega}_{0}\right\|_{L^{\infty}\left(\mathbb{R}^{2}\right)}\right)=\left(\|\omega(T)\|_{L^{1}\left(\mathbb{R}^{2}\right)}+\|\omega(T)\|_{L^{\infty}\left(\mathbb{R}^{2}\right)}\right) \leq\left(\left\|\omega_{0}\right\|_{L^{1}\left(\mathbb{R}^{2}\right)}+\left\|\omega_{0}\right\|_{L^{\infty}\left(\mathbb{R}^{2}\right)}\right)
$$

we see that $\tilde{T} \geq T$ and hence we can repeat this argument indefinitely, extending our solution for arbitrarily long times.

Thus, the only remaining step in the proof that we have unique global solutions for initial conditions in $X_{T}^{*}$ is to prove Lemma 3.46. We begin by showing that the $L^{1}\left(\mathbb{R}^{2}\right)$ norm of $\mathcal{N}$ is uniformly bounded.

$$
\begin{aligned}
\|\mathcal{N}(\tilde{\omega}, \omega)(t)\|_{L^{1}\left(\mathbb{R}^{2}\right)} & =\left\|\int_{0}^{t} e^{(t-s) \Delta} \nabla \cdot(\tilde{\mathbf{u}}(s) \omega(s)) d s\right\|_{L^{1}\left(\mathbb{R}^{2}\right)} \\
& \leq C \int_{0}^{t} \frac{1}{\sqrt{t-s}}\|\tilde{\mathbf{u}}(s) \omega(s)\|_{L^{1}\left(\mathbb{R}^{2}\right)} d s
\end{aligned}
$$

where the last inequality used Lemma 3.45 to bound the linear semigroup. By Hölder's inequality

$$
\|(\tilde{\mathbf{u}}(s) \omega(s))\|_{L^{1}\left(\mathbb{R}^{2}\right)} \leq\|\tilde{\mathbf{u}}(s)\|_{L^{4}\left(\mathbb{R}^{2}\right)}\|\omega(s)\|_{L^{4 / 3}\left(\mathbb{R}^{2}\right)},
$$

while Lemma 3.40 implies that $\left.\|\tilde{\mathbf{u}}(s)\|_{L^{4}\left(\mathbb{R}^{2}\right)} \leq C \| \tilde{\omega}(s)\right) \|_{L^{4 / 3}\left(\mathbb{R}^{2}\right)}$. Combining these estimates we find

$$
\|\mathcal{N}(\tilde{\omega}, \omega)(t)\|_{L^{1}\left(\mathbb{R}^{2}\right)} \leq C \int_{0}^{t} \frac{1}{\sqrt{t-s}}\|\tilde{\omega}(s)\|_{L^{4 / 3}\left(\mathbb{R}^{2}\right)}\|\omega(s)\|_{L^{4 / 3}\left(\mathbb{R}^{2}\right)} d s \leq C \sqrt{T}\|\tilde{\omega}\|_{*}\|\omega\|_{*} .
$$


A similar bound on the $L^{\infty}\left(\mathbb{R}^{2}\right)$ norm of $\mathcal{N}$ completes the proof. We again begin by using the bound in Lemma 3.45:

$$
\begin{aligned}
\|\mathcal{N}(\tilde{\omega}, \omega)(t)\|_{L^{\infty}\left(\mathbb{R}^{2}\right)} & =\left\|\int_{0}^{t} e^{(t-s) \Delta} \nabla \cdot(\tilde{\mathbf{u}}(s) \omega(s)) d s\right\|_{L^{\infty}\left(\mathbb{R}^{2}\right)} \\
& \leq C \int_{0}^{t} \frac{1}{\sqrt{t-s}}\|\tilde{\mathbf{u}}(s) \omega(s)\|_{L^{2}\left(\mathbb{R}^{2}\right)} d s
\end{aligned}
$$

But by Exercise 3.42 we have $\|\tilde{\mathbf{u}}(s)\|_{L^{\infty}\left(\mathbb{R}^{2}\right)} \leq\|\tilde{\omega}\|_{*}$ and by interpolation $\omega(s)\left\|_{L^{2}\left(\mathbb{R}^{2}\right)} \leq\right\| \tilde{\omega} \|_{*}$, hence

$$
\|\mathcal{N}(\tilde{\omega}, \omega)(t)\|_{L^{\infty}\left(\mathbb{R}^{2}\right)} \leq C \int_{0}^{t} \frac{1}{\sqrt{t-s}} d s\|\tilde{\omega}\|_{*}\|\omega\|_{*} .
$$

which completes the proof of Lemma 3.46 and concludes this section. 


\section{Lecture 4: The long-time asymptotics of solutions of the two-dimensional Navier-Stokes equation.}

In this final lecture we combine the methods developed in the first two lectures to describe the long-time behavior of solutions of the two-dimensional Navier-Stokes equation. We prove that any solution whose initial vorticity distribution is integrable will tend, as time goes to infinity, toward an Oseen vortex, a simple, explicitly computable solution of the NavierStokes equations in two-dimensions. We also give a detailed discussion of the long-time behavior of solutions whose total vorticity is small. The material in this lecture is largely joint work of Th. Gallay and myself and for more details the reader can consult the original papers $[7]$ and $[8]$.

Throughout this lecture we will consider the Navier-Stokes equation in the vorticity representation

$$
\begin{aligned}
& \frac{\partial \omega}{\partial t}=\Delta_{x} \omega-\mathbf{u} \cdot \nabla \omega \\
& \omega=\omega(x, t) \in \mathbb{R}, \quad x \in \mathbb{R}^{2}, t \geq 0 .
\end{aligned}
$$

where $\mathbf{u}$ is the velocity field associated with the vorticity $\omega$ via the Biot-Savart law. As discussed in the preceding lecture the vorticity formulation is particularly convenient in twodimensions where the vorticity is a scalar function. Furthermore as in Lecture 2 we will study solutions of $(61)$ in the weighted Hilbert spaces $L^{2}(m)$ and the vorticity has the advantage that if the initial vorticity distribution lies in one of these spaces the solution of (61) will remain in this space for all time, whereas that is not in general true of the velocity field. (This fact is not immediately apparent but is discussed and proven in [7].)

We begin, as we did in Lecture 2, by considering solutions of (61) in a neighborhood of the origin. Given the similarity between the vorticity equation and (9) we introduce scaling variables as we did in that case, namely we set:

$$
\begin{aligned}
& \omega(x, t)=\frac{1}{(1+t)} w\left(\frac{x}{\sqrt{1+t}}, \log (1+t)\right) \\
& \xi=\frac{x}{\sqrt{1+t}}, \quad \tau=\log (1+t)
\end{aligned}
$$

Note that this corresponds to taking the exponent $\alpha$ in (14) equal to $\alpha=d=2$. We still need to decide how to rescale the velocity field. Since the vorticity is a derivative of the velocity with respect to $x$, and since each $x$ derivative results in an extra factor of $\frac{1}{\sqrt{1+t}}$, this suggests that the velocity should scale as

$$
\mathbf{u}(x, t)=\frac{1}{\sqrt{1+t}} \mathbf{v}\left(\frac{x}{\sqrt{1+t}}, \log (1+t)\right) .
$$

Strong evidence that this is the "correct" scaling can be seen from the fact that with the rescaled velocity and vorticity fields defined by (62) and (63) $\mathbf{v}$ and $w$ are still related via 
the Biot-Savart law - namely:

$$
\mathbf{v}(\xi, \tau)=\frac{1}{2 \pi} \int_{\mathbb{R}^{2}} \frac{(x-y)^{\perp}}{|x-y|^{2}} w(\eta, \tau) d \eta,
$$

which we leave as an exercise for the reader to check.

Inserting (62) and (63) into (61) we find that

$$
\frac{\partial w}{\partial \tau}=\mathcal{L} w-\mathbf{v} \cdot \nabla w
$$

Here, $\mathcal{L}$ is the same operator that we studied in Lecture 2 - namely

$$
\mathcal{L} w=\Delta_{\xi} w+\frac{1}{2} \nabla_{\xi} \cdot(\xi w)
$$

Recall that the spectrum of $\mathcal{L}$ when acting on functions in $L^{2}(m)$ consists of the non-positive half integers, plus a half-plane of spectrum $\left\{\lambda \in \mathbb{C} \mid \Re(\lambda) \leq \frac{1}{2}-\frac{m}{2}\right\}$. Thus, for $m>1$ we expect that there will be a one-dimensional invariant manifold $W_{c}$, tangent at the origin to the eigenspace of the (simple) eigenvalue $\lambda=0$.

Remark 4.47 Verifying the hypotheses (H.1) - (H.4) of the (CHT) invariant manifold theorem requires combining the ideas of Lectures 2 and 3. Since the linear part of (62) is is the same as that of (34) verifying (H.1) and (H.2) is exactly the same as in Lecture 2. Verifying the hypotheses (H.3) and (H.4) on the nonlinearity follows from estimates very similar to those in Lecture 3 where we estimated the semi-group for (61) since the form of the nonlinear terms in (61) are the same as those in (65). In this case one must cut-off the nonlinear term outside a neighborhood of the origin in order to obtain the global estimates required in the (CHT) theorem, but that is again done in a fashion very similar to that in Lecture 2.

Let's next examine the motion on the manifold $W_{c}$. As in the case of the nonlinear heat equation in Lecture 2 a point on $W_{c}$ can be represented as

$$
w^{c}(\xi, \tau)=\alpha(\tau) \phi^{0}(\xi)+g\left(\alpha(\tau) \phi^{0}(\xi)\right)
$$

for some function $g: P_{0} L^{2}(m) \rightarrow Q_{0} L^{2}(m)$, where $P^{0}$ is the projection onto the eigenspace of $\lambda=0$ and $Q_{0}$ is the projection onto its complement. If we insert this form into (65) and apply the projection operator $P_{0}$ to both sides of the equation we find that

$$
\dot{\alpha}(\tau) \phi^{0}(\xi)=-P^{0}\left(\mathbf{v}^{c}(\xi, \tau) \cdot w(\xi, \tau)\right)
$$

where $\mathbf{v}^{c}$ is the velocity field associated to $w^{c}$ via the Biot-Savart Law. We now note two things:

(1.) $\left(P_{0} f\right)(\xi)=\left(\int_{\mathbb{R}^{2}} f(\xi) d \xi\right) \phi^{0}(\xi)$. 
(2.) The velocity field $\mathbf{v}^{c}$ is compressible (i.e. $\left.\nabla \cdot \mathbf{v}^{c}=0\right)$ and thus we can write $\mathbf{v}^{c}(\xi, \tau)$. $w(\xi, \tau)=\nabla \cdot\left(\mathbf{v}^{c}(\xi, \tau) w(\xi, \tau)\right)$.

But these two facts imply that

$$
P^{0}\left(\mathbf{v}^{c}(\xi, \tau) \cdot w(\xi, \tau)\right)=\left(\int_{\mathbb{R}^{2}} \nabla \cdot\left(\mathbf{v}^{c}(\xi, \tau) w(\xi, \tau)\right) d \xi\right) \phi^{0}(\xi)=0 .
$$

and hence that

$$
\dot{\alpha}(\tau)=0 .
$$

This implies that the center manifold consists entirely of fixed points! In fact, we can identify these fixed points more precisely. If one checks the velocity field corresponding (via the Biot-Savart) law to the vorticity field $\phi^{0}$ one finds that the velocity field is

$$
\mathbf{v}^{0}(x)=\frac{1}{2 \pi} \frac{x^{\perp}}{|x|^{2}}\left(1-e^{-|x|^{2} / 4}\right) .
$$

For the moment, the most important thing to note about this expression is that it is a purely tangential velocity field. As a consequence, since the vorticity $\phi^{0}$ depends only on $|x|$, the radial coordinate of $x$, we see that the nonlinear term in the vorticity equation:

$$
\mathbf{v}^{0}(x) \cdot\left(\nabla \phi^{0}\right)(x)=0 .
$$

Thus, since $\mathcal{L} \phi^{0}=0$ we see that the Gaussian vorticity distribution $\alpha \phi^{0}$ is a stationary solution of the rescaled vorticity equation (65). This family of solutions is known as the family of Oseen vortices.

Remark 4.48 Note that in the original, unrescaled variables, the Oseen vortices are not stationary solutions but rather spread and decay in the same way as does the fundamental solution of the heat equation.

Returning now to our discussion of the center manifold we know first of all, from the general theory of invariant manifolds discussed in Lecture 1 that all fixed points near the origin must lie in the center-manifold. Thus, for small $\alpha$ the family of Oseen vortices must be contained in the center-manifold. However, this is a one-dimensional family of solutions and the centermanifold itself is one-dimensional so in fact, the center-manifold in this case consists exactly of the family of Oseen vortices!

Again, appealing to the general theory of invariant manifolds we know that solutions near the origin will be attracted to one of the solutions on the center-manifold. In fact, we can determine which of the Oseen vortices is the limit by noting that the rescaled vorticity equation preserves the total vorticity - i.e. if $w(\xi, \tau)$ is the solution with initial condition $w_{0}(\xi)$ then

$$
\int_{\mathbb{R}^{2}} w(\xi, \tau) d \xi=\int_{\mathbb{R}^{2}} w_{0}(\xi) d \xi
$$

for all $\tau$. Thus, as $\tau$ goes to infinity, $w(\xi, \tau)$ approaches the vortex $\alpha \phi^{0}$ whose total vorticity is $\alpha=\int_{\mathbb{R}^{2}} w_{0}(\xi) d \xi$. More precisely we find 
Proposition 4.49 Fix $0<\mu<\frac{1}{2}$. There exist positive constants $r_{2}$ and $C$ such that for any initial data with $\left\|w_{0}\right\|_{2}<r_{2}$ the solution $w(\cdot, \tau)$ with initial conditions $w_{0}$ satisfies

$$
\left\|w(\cdot, \tau)-\alpha \phi^{0}(\cdot)\right\|_{2} \leq C e^{-\mu \tau}
$$

where $\alpha=\int_{\mathbb{R}^{2}} w_{0}(\xi) d \xi$.

By considering the invariant manifolds corresponding to other of the spectral subspaces, one can make other, more detailed statements about the asymptotics of small solutions. For instance, one thing that had been discovered about solutions of the Navier-Stokes equations was that certain relationships were required to hold between the spatial moments of solutions decaying with particular temporal rates [14]. However, the proofs of these moment conditions provided little insight into the meaning or origin of these relationships. In [7] Gallay and I showed that these moment conditions were the consequence of the requirement that the solution lie on certain invariant manifolds in the phase space and as a consequence were able to give a simple geometrical interpretation of the results on optimal decay rates. Additional uses and consequences of these sorts of invariant manifold theorems are contained in [7].

We turn now from the consideration of small solutions to a study of more general sorts of solutions of the two-dimensional Navier-Stokes equation. The first thing we note is that the Oseen vortices are not limited in size. The family of solutions

$$
\mathcal{O}^{\alpha}(\xi)=\alpha \phi^{0}(\xi)
$$

is an exact, stationary solution of (65) for all values of $\alpha$. Thus, we can extend the local center-manifold to a global manifold in this case. However, the proof that nearby solutions are attracted to the center manifold applies only to solutions of small norm, so our next task is to analyze the local stability of Oseen vortices of large magnitude.

Begin, by linearizing (65) about the vortex $\mathcal{O}^{\alpha}$. This leads to the linearized equation

$$
\frac{\partial w}{\partial \tau}=\mathcal{L} w-\alpha \Lambda w
$$

where the linear operator $\mathcal{L}$ is the one we studied in Lecture 2 and the operator $\Lambda$ is defined by:

$$
\Lambda w=\mathbf{v}^{0} \cdot \nabla w+\mathbf{v}^{w} \cdot \nabla \phi^{0}
$$

with $\mathbf{v}^{0}$ the velocity field associated to the vorticity $\phi^{0}$ and $\mathbf{v}^{w}$ the velocity field associated with the vorticity $w$.

We now consider the spectrum of the operator $\mathcal{L}-\alpha \Lambda$. The first observation is a bit of basic functional analysis. Note that operator $\Lambda$ is localized - i.e. the coefficient in each term of $\Lambda w$ decays as $|\xi| \rightarrow \infty$. Furthermore it is a first order differential operator while $\mathcal{L}$ is second order. These two facts taken together are sufficient to show that $\Lambda$ is a relatively compact perturbation of $\mathcal{L}$ and hence the essential spectrum of $\mathcal{L}$ and $\mathcal{L}-\alpha \Lambda$ must coincide. Thus we have 
Lemma 4.50 Fix $m>1$ and consider the operator $\mathcal{L}-\alpha \Lambda$ acting on its maximal domain in $L^{2}(m)$. Then

$$
\sigma_{\text {ess }}(\mathcal{L})=\sigma_{\text {ess }}(\mathcal{L}-\alpha \Lambda)=\left\{\lambda \in \mathbb{C} \mid \Re(\lambda) \leq \frac{1-m}{2}\right\}
$$

Remark 4.51 More details on the proof of this lemma and succeeding results in this lecture can be found in [8].

As a consequence of Lemma 4.50 the stability or instability of the Oseen vortices of large norm will be determined by whether or not the isolated eigenvalues of $\mathcal{L}-\alpha \Lambda$ lie in the left or right half plane. One of these eigenvalues can be immediately and explicitly computed and we find:

Lemma 4.52 The operator $\mathcal{L}-\alpha \Lambda$ has an eigenvalue $\lambda=0$ with eigenfunction $\phi^{0}$ for all values of $\alpha$.

Since the projection of a function $f$ onto this eigenspace is just given by the product of $\phi^{0}$ with the integral of $f$, the complementary subspace to the zero eigenspace consists of the functions of zero mean. Thus, we can restrict our attention of the space of functions $L_{0}^{2}(m)=\left\{f \in L^{2}(m) \mid \int_{\mathbb{R}^{2}} f(\xi) d \xi=0\right\}$. When restricted to this space we have the following result:

Proposition 4.53 Fix $m>1$ and $\alpha \in \mathbb{R}$. Then any eigenvalue of $\lambda$ of $\mathcal{L}-\alpha \Lambda$ with eigenfunction in $L_{0}^{2}(m)$ satisfies

$$
\Re(\lambda) \leq \max \left(-\frac{1}{2}, \frac{1-m}{2}\right)
$$

Remark 4.54 Note that this proposition, in combination with the above remark about the zero eigenvalue and the essential spectrum implies that the Oseen vortices are spectrally stable for all values of $\alpha$. Given this spectral information it follows in a fairly straighforward fashion that the Oseen vortices are locally stable for all values of $\alpha$-namely given an initial condition of (65) sufficiently close to an Oseen vortex the resulting solution of the vorticity equation will converge to an Oseen vortex as time tends toward infinity.

Remark 4.55 Because we have scaled all other physical parameters to have value one, $\alpha$ can be thought of as the Reynolds number for the problem. Thus, in contrast to many other fluid mechanical situations increasing the Reynolds number in this problem does not lead to instability. In fact, numerical computations [15] indicate that the real parts of most eigenvalues of $\mathcal{L}-\alpha \Lambda$ actually become more negative as $\alpha$ increases so that the increasing Reynolds number actually has a sort of stabilizing effect. 
The proof of Proposition 4.53 consists of three steps:

(1.) By writing out the eigenvalue equation in polar coordinates a straightforward but complicated analysis shows that regardless of the value of $\alpha$ any eigenfunction in $L_{0}^{2}(m)$ whose real part is larger than $\frac{1-m}{2}$ must have Gaussian decay as $|\xi| \rightarrow \infty$. Thus the eigenfunctions are very strongly localized in space, regardless of the value of $\alpha$. Given these results we define a new Hilbert space $X=\left\{w \in L^{2}\left(\mathbb{R}^{2}\right) \mid w / \sqrt{\phi^{0}} \in L^{2}\left(\mathbb{R}^{2}\right)\right\}$, equipped with the innerproduct

$$
\left(w_{1}, w_{2}\right)_{X}=\int_{\mathbb{R}^{2}} \frac{\bar{w}_{1}(\xi) w_{2}(\xi)}{\phi^{0}(\xi)} d \xi .
$$

We know that the eigenfunctions of $\mathcal{L}-\alpha \Lambda$ lie in $X$ and (since we can continue to ignore the eigenvalue zero) we will study the spectrum on the space $X_{0}=X \cap L_{0}^{2}(m)$.

(2.) We next compute the representation of $\mathcal{L}$ in the Hilbert space $X$ which is given by

$$
\mathcal{L}^{X}=\left(\phi^{0}\right)^{(-1 / 2)} \mathcal{L}\left(\phi^{0}\right)^{(1 / 2)}=\Delta_{\xi}-\frac{|\xi|^{2}}{16}+\frac{1}{2} .
$$

This operator is the well known quantum mechanical oscillator and as is well known in quantum mechanics:

(a) $\mathcal{L}^{X}$ is self-adjoint.

(b) The spectrum of $\mathcal{L}^{X}$ consists only of the eigenvalues $-n / 2, n=0,1,2, \ldots$.

The second of these points is not surprising but the fact that $\mathcal{L}$ is self-adjoint in the Hilbert space $X$ will be critical in what follows.

(3.) The final point is the computation of the representation of $\Lambda$ in $X_{0}$. Writing out the expression for $\Lambda$ in the $X$-inner product one finds:

$$
(\tilde{w}, \Lambda w)_{X}=\int_{\mathbb{R}^{2}}\left(\frac{1}{\phi^{0}} \tilde{w} \mathbf{v}^{0} \cdot \nabla w-\frac{1}{2} \tilde{w}(\mathbf{v} \cdot \xi)\right) d \xi,
$$

where we used the fact that $\nabla \phi^{0}=-\frac{\xi}{2} \phi^{0}$. Two easy calculations show that

$$
\int_{\mathbb{R}^{2}}\left(\frac{1}{\phi^{0}} \tilde{w}\right) \mathbf{v}^{0} \cdot \nabla w d \xi=-\int_{\mathbb{R}^{2}} \frac{1}{\phi^{0}} w \mathbf{v}^{0} \cdot \nabla \tilde{w} d \xi .
$$

and

$$
\tilde{w}(\mathbf{v} \cdot \xi)+w(\tilde{\mathbf{v}} \cdot \xi)=\left(\xi_{1} \partial_{1}-\xi_{2} \partial_{2}\right)\left(v_{1} \tilde{v}_{2}+v_{2} \tilde{v}_{1}\right)+\left(\xi_{1} \partial_{2}+\xi_{2} \partial_{1}\right)\left(v_{2} \tilde{v}_{2}-v_{1} \tilde{v}_{1}\right) .
$$

Integrating both sides of the second equation in (73) we see that

$$
\int_{\mathbb{R}^{2}} \tilde{w}(\mathbf{v} \cdot \xi)+w(\tilde{\mathbf{v}} \cdot \xi) d \xi=0
$$

which when combined with (72) and (73) imply that

$$
(\tilde{w}, \Lambda w)_{X}=-(\Lambda \tilde{w}, w)_{X}
$$

or 
Lemma 4.56 The linear operator $\Lambda$ is skew-symmetric on $X_{0}$.

Proposition 4.53 now follows from the following property from linear algebra. Namely, suppose that $\mathcal{L}$ is a self-adjoint operator on a Hilbert space $X_{0}$ whose spectrum lies in the half line $\lambda \leq-\mu<0$. Then if $\Lambda$ is skew-adjoint on $X_{0}$ any eigenvalue of $\mathcal{L}-\alpha \Lambda$ has real part less than equal or equal to $\mu$. To see why this is so, suppose that

$$
(\mathcal{L}-\alpha \Lambda) \phi=\lambda \phi .
$$

Then

$$
\begin{aligned}
\lambda(\phi, \phi)_{X_{0}} & =(\phi, \mathcal{L} \phi)_{X_{0}}-\alpha(\phi, \Lambda \phi)_{X_{0}}, \quad \text { while } \\
\bar{\lambda}(\phi, \phi)_{X_{0}} & =\overline{(\phi, \mathcal{L} \phi)_{X_{0}}}-\alpha \overline{(\phi, \Lambda \phi)_{X_{0}}}=(\mathcal{L} \phi, \phi)_{X_{0}}-\alpha(\Lambda \phi, \phi)_{X_{0}} \\
& =(\phi, \mathcal{L} \phi)_{X_{0}}+\alpha(\phi, \Lambda \phi)_{X_{0}}
\end{aligned}
$$

Adding these two expressions together yields

$$
\Re(\lambda)=(\phi, \mathcal{L} \phi)_{X_{0}} \leq-\mu .
$$

Reviewing the picture we have of solutions of the two-dimensional Navier-Stokes equation so far we see that we have a global center manifold, consisting of the family of Oseen vortices which are locally stable for all values of $\alpha$. The final question that we consider is the behavior of solutions of (65) for arbitrary initial data (i.e. for initial vorticity distributions which are not close to one of the Oseen vortices.)

Given the results of Lecture 3 it is natural to require that the initial vorticity distribution be in $L^{1}\left(\mathbb{R}^{2}\right)$. We know that the solution with this initial condition exists for all time and thus we can ask what its $\omega$-limit set is. From the first lecture we know that in order to be sure that the $\omega$-limit set exists we need to check whether the trajectory remains in a compact subset of $L^{1}\left(\mathbb{R}^{2}\right)$. The details needed to establish this fact are presented in [8] but we note two main ideas are that by Rellich's criterion subspaces of $L^{1}\left(\mathbb{R}^{2}\right)$ that have some smoothness and decay at infinity are compact. In our problem:

- smoothness comes from the smoothing properties of the semigroup which are preserved by the nonlinearity.

- decay at infinity comes from estimates of the solution of the vorticity equation due to Carlen and Loss [3].

Given that the $\omega$-limit set exists how can we calculate it? We determine the $\omega$-limit set with the aid of two Lypunov functions:

1. The first tells us that the $\omega$-limit set consists of functions that do not change sign - i.e. an element of the $\omega$-limit set of a solution with initial value $w_{0}$ is either everywhere non-positive or everywhere non-negative. 
2. The second will identify those positive (or negative) functions that can be part of the $\omega$-limit set.

Lyapunov Function No. 1: This Lyapunov function is closely related to Example 1.16 from Lecture 1. Define

$$
\Phi(w(\tau))=\int_{\mathbb{R}^{2}}|w(\xi, \tau)| d \xi .
$$

One then has:

Lemma 4.57 Let $w_{0} \in L^{1}\left(\mathbb{R}^{2}\right)$ and let $w$ be the solution of the rescaled vorticity equation with this initial condition. Then $\Phi(w(\tau)) \leq \Phi\left(w_{0}\right)$ for all $\tau \geq 0$. Moreover, equality holds if and only if $w_{0} \in \Sigma$ where

$$
\Sigma=\left\{w \in L^{1}\left(\mathbb{R}^{2}\right)\left|\int_{\mathbb{R}^{2}}\right| w(\xi)|d \xi=| \int_{\mathbb{R}^{2}} w(\xi) d \xi \mid\right\} .
$$

Proof: This lemma follows from the maximum principle very much along the lines of Example 1.16. Indeed that example established this result for the linear terms in (65). Including the nonlinear terms in the equation causes no essential difficulty and we leave the details of this argument as an exercise for the reader.

Note that as a corollary of this lemma and the LaSalle Invariance Principle we have

Corollary 4.58 Let $w_{0} \in L^{1}\left(\mathbb{R}^{2}\right)$ The $\omega$-limit set of the solution with this initial condition must lie in $\Sigma$.

Lyapunov Function No. 2: Since from the preceeding corollary the $\omega$-limit set is contained in set of positive (or negative) functions our second Lyapunov function will be defined only on such functions. This second Lyapunov function is motivated by Lyapunov functions used in kinetic theory where one also wants to prove the convergence of solutions toward Gaussian profiles and is known in that field as the relative entropy function. Define $\Sigma_{+}=$ $\{w \in \Sigma \mid w(\xi) \geq 0$ almost everywhere $\}$ and define $H: \Sigma_{+} \cap L^{2}(m) \rightarrow \mathbb{R}$ by

$$
H(w(\tau))=\int_{\mathbb{R}^{2}} w(\xi, \tau) \log \left(\frac{w(\xi, \tau)}{\phi^{0}(\xi)}\right) d \xi .
$$

If $m>3$ the functions $w$ decay fast enough at infinity that one can show:

1. $H$ is defined and continuous on $\Sigma_{+} \cap L^{2}(m)$

2. $H$ is bounded below by $-1 / e$. 
Even more importantly for our purposes, $H$ is decreasing along trajectories and hence a Lyapunov function. Assume for the moment that $w$ is smooth enough that we can differentiate $H(w(\tau))$ by pulling the derivative through the integral sign. (The general case can be handled by approximation by smooth functions.) Then

$$
\frac{d}{d \tau} H(w(\tau))=\int_{\mathbb{R}^{2}}\left(1+\log \frac{w}{\phi^{0}}\right) \partial_{\tau} w d \xi=\int_{\mathbb{R}^{2}}\left(1+\log \frac{w}{\phi^{0}}\right)(\mathcal{L} w-\mathbf{v} \cdot \nabla w) d \xi .
$$

We break this last integral into two pieces and consider each piece separately. First note that thanks to the special properties of the Gaussian

$$
\mathcal{L} w=\operatorname{div}\left(\phi^{0} \nabla\left(\frac{w}{\phi^{0}}\right)\right)
$$

so that

$$
\int_{\mathbb{R}^{2}}\left(1+\log \frac{w}{\phi^{0}}\right)(\mathcal{L} w) d \xi=-\int_{\mathbb{R}^{2}} \phi^{0}\left(\nabla\left(\log \frac{w}{\phi^{0}}\right)\right) \cdot \nabla\left(\frac{w}{\phi^{0}}\right)=-\int_{\mathbb{R}^{2}} w\left|\nabla\left(\log \frac{w}{\phi^{0}}\right)\right|^{2} d \xi
$$

To treat the second term in (76) we first integrate by parts to obtain

$$
\begin{array}{r}
-\int_{\mathbb{R}^{2}}\left(1+\log \frac{w}{\phi^{0}}\right)(\mathbf{v} \cdot \nabla w)=-\int_{\mathbb{R}^{2}}\left(1+\log \frac{w}{\phi^{0}}\right)(\nabla \cdot(\mathbf{v} w)) d \xi \\
=\int_{\mathbb{R}^{2}} \phi^{0} \mathbf{v} \cdot \nabla\left(\frac{w}{\phi^{0}}\right) d \xi=\int_{\mathbb{R}^{2}} \mathbf{v} \cdot \nabla w d \xi-\frac{1}{2} \int_{\mathbb{R}^{2}}(\xi \cdot \mathbf{v}) w d \xi .
\end{array}
$$

We claim finally that each of these two last integrals vanish. For the first, this is obvious since $\mathbf{v} \cdot \nabla w=\nabla \cdot(\mathbf{v} w)$. For the second note that $w=\partial_{\xi_{1}} v_{2}-\partial_{\xi_{2}} v_{1}\left(\right.$ where $\left.\mathbf{v}=\left(v_{1}, v_{2}\right)\right)$ and hence

$$
\begin{aligned}
& \int_{\mathbb{R}^{2}}(\xi \cdot \mathbf{v}) w d \xi=\int_{\mathbb{R}^{2}}\left(\xi_{1} v_{1}+\xi_{2} v_{2}\right)\left(\partial_{\xi_{1}} v_{2}-\partial_{\xi_{2}} v_{1}\right) d \xi \\
& \quad=\int_{\mathbb{R}^{2}} \xi_{1} v_{1} \partial_{\xi_{1}} v_{2} d \xi+\int_{\mathbb{R}^{2}} \xi_{2} v_{2} \partial_{\xi_{1}} v_{2} d \xi-\int_{\mathbb{R}^{2}} \xi_{1} v_{1} \partial_{\xi_{2}} v_{1} d \xi-\int_{\mathbb{R}^{2}} \xi_{2} v_{2} \partial_{\xi_{2}} v_{1} d \xi
\end{aligned}
$$

Note that the second and third of these integrals vanish since the second can be rewritten as $\frac{1}{2} \int_{\mathbb{R}^{2}} \partial_{\xi_{1}}\left(\xi_{2}\left(v_{2}\right)^{2}\right) d \xi=0$ and analogously for the third. In the first and fourth integrals we integrate by parts to obtain

$$
\begin{gathered}
-\int_{\mathbb{R}^{2}} v_{1} v_{2} d \xi-\int_{\mathbb{R}^{2}} \xi_{1}\left(\partial_{\xi_{1}} v_{1}\right) v_{2} d \xi+\int_{\mathbb{R}^{2}} v_{1} v_{2} d \xi+\int_{\mathbb{R}^{2}} \xi_{2}\left(\partial_{\xi_{2}} v_{2}\right) v_{1} d \xi \\
=\int_{\mathbb{R}^{2}} \xi_{1}\left(\partial_{\xi_{2}} v_{2}\right) v_{2} d \xi-\int_{\mathbb{R}^{2}} \xi_{2}\left(\partial_{\xi_{1}} v_{1}\right) v_{1} d \xi=0
\end{gathered}
$$

where the next to last equality used the fact that $\mathbf{v}$ is incompressible and the final equality noted that the first integral could be written as $\frac{1}{2} \int_{\mathbb{R}^{2}} \partial_{\xi_{2}}\left(\xi_{1}\left(v_{2}\right)^{2}\right) d \xi=0$ and similarly for the second. 
Remark 4.59 In fact, one needs to take a little more care with this calculation since for general velocity fields $\mathbf{v}$, integrals like $\int_{\mathbb{R}^{2}} \xi_{2} v_{2} \partial_{\xi_{1}} v_{2} d \xi$ may fail to converge due to the relatively slow decay at infinity of the velocity field. Nonetheless, the entire expression $\int_{\mathbb{R}^{2}}(\xi \cdot \mathbf{v}) w d \xi$ is convergent because of cancellations between various terms. The easiest way to take advantage of these cancellations is to rewrite the velocity in terms of the vorticity via the Biot-Savart law and then argue that the integral must vanish by symmetry. (See [8] for details.) However, I think that the present argument which works entirely with the velocity field gives somewhat more intuition into why these terms vanish.

Putting these computations together we see that we have shown:

\section{Lemma 4.60}

$$
\frac{d}{d \tau} H(w(\tau))=-\int_{\mathbb{R}^{2}} w\left|\nabla\left(\log \frac{w}{\phi^{0}}\right)\right|^{2} d \xi
$$

This lemma implies that the Lyapunov function $H$ is strictly decreasing unless $w$ is a multiple of the Gaussian $\phi^{0}$ and implies, as an immediate corollary:

Corollary 4.61 Assume that $w_{0} \in L^{2}(m) \cap \Sigma$ with $m>3$. The $H(w(\tau)) \leq H\left(w_{0}\right)$ for all points $w(\tau)$ in the forward orbit of $w_{0}$ and $H(w(\tau))=H\left(w_{0}\right)$ for all $\tau \geq 0$ if and only if $w_{0}=\alpha G$ for some $\alpha \geq 0$.

We can now put together the various pieces of this argument to derive a quite complete picture of the long-time asymptotic behavior of solutions of the two-dimensional NavierStokes equations. Suppose we consider any solution of (65) whose initial vorticity $w_{0} \in L^{2}(m)$ with $m>3$. By Lemma 4.57 we know that any point $w^{*}$ in the omega limit set of $w_{0}$ must lie in the set $\Sigma$ of functions which do not change sign. Assume, without loss of generality, that $w^{*}(\xi)>0$.

From the general theory of Lyapunov functionals we know that the solution of the vorticity equation with initial conditions $w^{*}$ exists for all time $t \in \mathbb{R}$. Combining this observation with Corollary 4.61 implies that the orbit of $w^{*}$ consists of the single point $\alpha_{0} G$ where $\alpha_{0}=\int w_{0}(\xi) d \xi$ and hence that the omega-limit set of any point $w_{0} \in L^{2}(m)$ with $m>3$ consists of the Oseen vortex with the same total vorticity.

In fact, using results of Carlen and Loss [3] on the spatial decay rate of solutions of the two-dimensional vorticity equation one can prove that any point in the omega-limit set of a solution whose initial vorticity is in $L^{1}\left(\mathbb{R}^{2}\right)$ must lie in $L^{2}(m)$ for all $m>1$ - in particular it must lie in $L^{2}(m)$ for some $m>3$. Then, proceeding as above, we find that the omega-limit set must again consist just of an Oseen vortex. If we now undo the change of variables (62) and (63) we see that solutions $\omega(x, t)$ satisfy: 
Theorem 4.62 If $\omega_{0} \in L^{1}\left(\mathbb{R}^{2}\right)$, the solution $\omega(x, t)$ of (61) satisfies

$$
\lim _{t \rightarrow \infty} t^{1-\frac{1}{p}}\left|\omega(\cdot, t)-\frac{\alpha}{t} G\left(\frac{\cdot}{\sqrt{t}}\right)\right|_{p}=0, \text { for } 1 \leq p \leq \infty,
$$

where $\alpha=\int_{\mathbb{R}^{2}} \omega_{0}(x) x$. If $\mathbf{u}(x, t)$ is the solution of the two-dimensional Navier-Stokes equation obtained from $\omega(x, t)$ via the Biot-Savart law, then

$$
\lim _{t \rightarrow \infty} t^{\frac{1}{2}-\frac{1}{q}}\left|\mathbf{u}(\cdot, t)-\frac{\alpha}{\sqrt{t}} \mathbf{v}^{G}\left(\frac{\cdot}{\sqrt{t}}\right)\right|_{q}=0, \text { for } 2<q \leq \infty .
$$

where $\mathbf{v}^{G}$ is the velocity field (68) associated to the Oseen vortex.

\subsection{Conclusions}

Summing up, we see that the dynamical systems method provides a quite complete view of the long-time asymptotics of general solutions of the two-dimensional Navier-Stokes or vorticity equations.

While one cannot hope to obtain comparably complete information about solutions of the three-dimensional Navier-Stokes equation where even the existence of solutions with general initial data is unproven it turns out that one can use the ideas developed above to understand the existence and stability of some classes of vortex solutions related to the Burgers vortices, an explicit family of solutions of the three-dimensional Navier-Stokes equations believed to be important for turbulent flows. [9], [10].

Another interesting and open question is to unterstand the intermediate time behavior of solutions of the two-dimensional Navier-Stokes equation. While the results proven above imply that eventually one converges to a single vortex solution, numerical simulations imply that the evolution at intermediate time scales is dominated by the interaction and merger of pairs of vortices. A better understanding of this merger process would be very intereresting and also have important applications.

\section{References}

[1] Peter W. Bates and Christopher K. R. T. Jones. Invariant manifolds for semilinear partial differential equations. In Dynamics reported, Vol. 2, volume 2 of Dynam. Report. Ser. Dynam. Systems Appl., pages 1-38. Wiley, Chichester, 1989.

[2] Matania Ben-Artzi. Global solutions of two-dimensional Navier-Stokes and Euler equations. Arch. Rational Mech. Anal., 128(4):329-358, 1994.

[3] Eric A. Carlen and Michael Loss. Optimal smoothing and decay estimates for viscously damped conservation laws, with applications to the 2-D Navier-Stokes equation. Duke Math. J., 81(1):135-157 (1996), 1995. A celebration of John F. Nash, Jr. 
[4] Xu-Yan Chen, Jack K. Hale, and Bin Tan. Invariant foliations for $C^{1}$ semigroups in Banach spaces. J. Differential Equations, 139(2):283-318, 1997.

[5] Charles R. Doering and J. D. Gibbon. Applied analysis of the Navier-Stokes equations. Cambridge Texts in Applied Mathematics. Cambridge University Press, Cambridge, 1995.

[6] Isabelle Gallagher and Thierry Gallay. Équations de la mechanique des fluides. http://www-fourier.ujf-grenoble.fr/ECOLETE/ecole2005/Note-Gallagher.pdf, 2006.

[7] Thierry Gallay and C. Eugene Wayne. Invariant manifolds and the long-time asymptotics of the Navier-Stokes and vorticity equations on $\mathbf{R}^{2}$. Arch. Ration. Mech. Anal., 163(3):209-258, 2002.

[8] Thierry Gallay and C. Eugene Wayne. Global stability of vortex solutions of the twodimensional Navier-Stokes equation. Comm. Math. Phys., 255(1):97-129, 2005.

[9] Thierry Gallay and C. Eugene Wayne. Three-dimensional stability of Burgers vortices: the low Reynolds number case. Phys. D, 213(2):164-180, 2006.

[10] Thierry Gallay and C. Eugene Wayne. Existence and stability of asymmetric Burgers vortices. J. Math. Fluid Mech., 9(2):243-261, 2007.

[11] Daniel Henry. Geometric theory of semilinear parabolic equations, volume 840 of Lecture Notes in Mathematics. Springer-Verlag, Berlin, 1981.

[12] J. P. LaSalle. The stability of dynamical systems. Society for Industrial and Applied Mathematics, Philadelphia, Pa., 1976. With an appendix: "Limiting equations and stability of nonautonomous ordinary differential equations" by Z. Artstein, Regional Conference Series in Applied Mathematics.

[13] Alexander Mielke. Locally invariant manifolds for quasilinear parabolic equations. Rocky Mountain J. Math., 21(2):707-714, 1991. Current directions in nonlinear partial differential equations (Provo, UT, 1987).

[14] T. Miyakawa and M. Schonbek. On optimal decay rates for weak solutions of the navierstokes equations in $\mathbb{R}^{n}$. In Proceedings of Partial Differential Equations and Applications (Olomouc, 1999), volume 126, pages 443-455. 2001.

[15] A. Prochazka and D. I. Pullin. On the two-dimensional stability of the axisymmetric Burgers vortex. Phys. Fluids, 7(7):1788-1790, 1995.

[16] A. Vanderbauwhede and G. Iooss. Center manifold theory in infinite dimensions. In Dynamics reported: expositions in dynamical systems, volume 1 of Dynam. Report. Expositions Dynam. Systems (N.S.), pages 125-163. Springer, Berlin, 1992. 